\title{
Lie-Schwinger block-diagonalization and gapped quantum chains: analyticity of the ground-state energy
}

\author{
S. Del Vecchio*, J. Fröhlich ${ }^{\dagger}$, A. Pizzo ${ }^{\ddagger}$, S. Rossi ${ }^{\S}$
}

$17 / 08 / 2019$

\begin{abstract}
We consider quantum chains whose Hamiltonians are perturbations by interactions of short range of a Hamiltonian that does not couple the degrees of freedom located at different sites of the chain and has a strictly positive energy gap above its ground-state energy. For interactions that are form-bounded w.r.t. the on-site Hamiltonian terms, we have proven that the spectral gap of the perturbed Hamiltonian above its ground-state energy is bounded from below by a positive constant uniformly in the length of the chain, for small values of a coupling constant; see [DFPR]. The main result of this paper is that, under the same hypotheses, the ground-state energy is analytic for values of the coupling constant belonging to a fixed interval, uniformly in the length of the chain. Furthermore, assuming that the interaction potentials are invariant under translations, we prove that, in the thermodynamic limit, the energy per site is analytic for values of the coupling constant in the same fixed interval. In our proof we use a new method introduced in [FP], which is based on local Lie-Schwinger conjugations of the Hamiltonians associated with connected subsets of the chain. We prove a rather strong result concerning complex Hamiltonians corresponding to complex values of the coupling constant.
\end{abstract}

\section{Introduction: Models and Results}

In this paper, we continue our study of spectral properties of Hamiltonians associated with a family of quantum chains with interactions of short range. Included are bosonic systems such as an array of coupled anharmonic oscillators. For the Hamiltonians considered in this paper, we have proven that their ground-state energy is finitely degenerate and the spectral gap above the ground-state energy is bounded from below by a positive constant, uniformly in the length of the chain; see [DFPR]. The purpose of the present paper is to show that, under the hypotheses considered in [DFPR], the ground-state energy is actually analytic in the coupling

\footnotetext{
*Dipartimento di Matematica, Università di Roma “Tor Vergata", Italy / email: delvecch@ mat.uniroma2.it

†Institut für Theoretiche Physik, ETH-Zürich , Switzerland / email: juerg@ phys.ethz.ch

"Dipartimento di Matematica, Università di Roma “Tor Vergata", Italy / email: pizzo@ mat.uniroma2.it

§Dipartimento di Matematica, Università di Roma “Tor Vergata", Italy / email: rossis@ mat.uniroma2.it
} 
constant, in a fixed disk centered at the origin that can be chosen to be independent of $N$ (number of sites in the chain). The method ${ }^{1}$ is based on iterative conjugations of the Hamiltonians, which serve to block-diagonalise them with respect to a fixed orthogonal projection and its orthogonal complement, and that carries over to the complex Hamiltonians obtained from the physical ones by replacing the physical (real) coupling constant by a complex parameter.

Our analysis is motivated by recent widespread interest in characterising topological phases of matter; see, e.g., [MN], [NSY], [BN].

The type of Hamiltonians considered in this paper have been studied before to prove the existence of a spectral gap above the ground-state energy (or the existence of an isolated eigenvalue for complex Hamiltonians), often using so-called "cluster expansions", but usually for bounded and real interactions; see [DFF], [FFU], [KT], [Y], [KU], [DS] [H] and refs. given there. Regarding the main result of this paper, i.e., analyticity of the ground-state energy, we mention that, in the paper by D. Yarotsky (see [Y]) dealing with unbounded interactions, a weak*-analyticity result was proven: For any local observable (i.e., an operator of bounded "lattice support"), its expectation value in the ground state of the system is analytic in the coupling constant, $t$, in a disk independent of the size of the lattice.

\subsection{A concrete family of quantum chains}

The Hilbert space of pure state vectors of the quantum chains studied in this paper has the form

$$
\mathcal{H}^{(N)}:=\bigotimes_{j=1}^{N} \mathcal{H}_{j}
$$

where $\mathcal{H}_{j} \simeq \mathcal{H}, \forall j=1,2, \ldots, N$ and where $\mathcal{H}$ is a separable Hilbert space. Let $H$ be a non-negative operator with the properties that 0 is an eigenvalue of $H$ corresponding to an eigenvector $\Omega \in \mathcal{H}$, and

$$
H \Upsilon_{\{\mathbb{C} \Omega\}^{\perp}} \geq \mathbb{1}
$$

where $\mathbb{1}$ is the identity operator.

We define

$$
H_{i}:=\mathbb{1}_{1} \otimes \cdots \otimes \underset{\substack{\uparrow \\ i^{t} h_{\text {slot }}}}{H} \otimes \ldots \mathbb{1}_{N} .
$$

By $P_{\Omega_{i}}$ we denote the orthogonal projector onto the subspace

$$
\left.\mathcal{H}_{1} \otimes \cdots \otimes \underset{\substack{\uparrow \\ \uparrow}}{\mathbb{C} \Omega}\right\}, \otimes \otimes \mathcal{H}_{N} \subset \mathcal{H}^{(N)}, \quad \text { and } \quad P_{\Omega_{i}}^{\perp}:=\mathbb{1}-P_{\Omega_{i}} .
$$

Then

with

$$
H_{i}=P_{\Omega_{i}} H_{i} P_{\Omega_{i}}+P_{\Omega_{i}}^{\perp} H_{i} P_{\Omega_{i}}^{\perp},
$$

$$
P_{\Omega_{i}} H_{i} P_{\Omega_{i}}=0, \quad P_{\Omega_{i}}^{\perp} H_{i} P_{\Omega_{i}}^{\perp} \geq P_{\Omega_{i}}^{\perp} .
$$

In [DFPR] we have studied quantum chains on the graph $I_{N-1,1}:=\{1, \ldots, N\}, N<\infty$ arbitrary, with Hamiltonians of the form

$$
K_{N} \equiv K_{N}(t):=\sum_{i=1}^{N} H_{i}+t \sum_{\substack{I_{k, i} \subset I_{N-1,1} \\ k \leq \bar{k}}} V_{I_{k, i}}
$$

\footnotetext{
${ }^{1}$ See [DFFR] for the use of a similar block-diagonalization in a simpler context. Ideas somewhat similar to the scheme in [FP] have been used in work of J. Z. Imbrie, [I1], [I2].
} 
where $t \in \mathbb{R}$ is a coupling constant, $\bar{k}<\infty$ is an arbitrary, but fixed integer, $I_{k, i}$ is the "interval" given by $\{i, \ldots, i+k\}, i=1, \ldots, N-k$, and $V_{I_{k, i}}$ is a symmetric operator acting on $\mathcal{H}^{(N)}$ with the property that

$$
V_{I_{k, i}} \text { acts as the identity on } \bigotimes_{j \in I_{N-1,1}, j \notin I_{k, i}} \mathcal{H}_{j}
$$

We call $I_{k, i}$ the "support" of $V_{I_{k, i}}$. It is assumed that

$$
D\left(\left(H_{I_{k, i}}^{0}\right)^{\frac{1}{2}}\right) \subseteq D\left(V_{I_{k, i}}\right), \quad\left|\left\langle\phi, V_{I_{k, i}} \phi\right\rangle\right| \leq a\left\langle\phi,\left(H_{I_{k, i}}^{0}+1\right) \phi\right\rangle,
$$

for any $\phi \in D\left(\left(H_{I_{k, i}}^{0}\right)^{\frac{1}{2}}\right)$ where $H_{I_{k, i}}^{0}:=\sum_{l=i}^{i+k} H_{l}$, for some universal constant $a>0$. Under these assumptions, and using the inequality

$$
\sum_{I_{k, i} \subset I_{N-1,1}} H_{I_{k, i}}^{0} \leq(k+1) \sum_{i=1}^{N} H_{i},
$$

one shows that, for $|t|$ sufficiently small (depending on $\bar{k}$ and $a$, but independent of $N$ ), the symmetric operator in (1.5) is defined and bounded from below on $D\left(H_{I_{N-1,1}^{0}}^{0}\right)$. It can be extended to a densely defined self-adjoint operator whose domain we denote $D\left(K_{N}\right) \subseteq D\left(\left(H_{I_{N-1,1}}^{0}\right)^{\frac{1}{2}}\right)$, namely the Friedrichs extension, which is uniquely determined by the property $D\left(K_{N}\right) \subseteq$ $D\left(\left(H_{I_{N-1,1}}^{0}\right)^{\frac{1}{2}}\right) \equiv Q\left(K_{N}\right)$, where $Q\left(K_{N}\right)$ is the form domain. It is not difficult to check that, under our hypotheses on the potentials, this extension coincides with the self-adjoint operator defined through the KLMN theorem, starting from the closed quadratic form associated with (1.5).

In the present paper, we will consider a complex coupling constant, $\tau$, instead of $t \in \mathbb{R}$, and analyze the closed quadratic form given by

$$
\sum_{i=1}^{N} H_{i}+\tau \sum_{\substack{I_{k, i} \subset I_{N-1,1} \\ k \leq \bar{k}}} V_{I_{k, i}}:=\kappa_{N} \equiv \kappa_{N}(\tau)
$$

For $|\tau|$ sufficiently small (depending on $\bar{k}$ and $a$, but independent of $N$ ), we have the following general results (see Theorems 3.9 and 2.1 of $[\mathrm{K}]$ ), starting from the closed form defined in (1.9):

i) there is a domain denoted by $D\left(K_{N}\right) \subset Q\left(\kappa_{n}\right)$, where an m-sectorial - and thus closed - operator $K_{N} \equiv K_{N}(\tau)$ is defined and the associated form coincides with $\kappa_{N}(\tau)$ (the operator $K_{N}$ is uniquely determined by the properties in i));

ii) the form domain $Q\left(\kappa_{N}\right)$ coincides with the form domain, $Q\left(H_{I_{N-1,1}}^{0}\right) \equiv D\left(\left(H_{I_{N-1,1}}^{0}\right)^{\frac{1}{2}}\right)$, of $H_{I_{N-1,1}^{0}}^{0}$.

Hence, we deduce that $\left.D\left(K_{N}\right)\right) \subseteq D\left(\left(H_{I_{N-1,1}}^{0}\right)^{\frac{1}{2}}\right)$. Furthermore, $D\left(K_{N}\right)$ is dense, since $m$ sectorial operators are densely defined. From [K, Corollary 2.4, p. 323] we get that $D\left(H_{I_{N-1,1}}^{0}\right) \subseteq$ $D\left(K_{N}\right)$; in fact, this follows from the following statements:

1) the l-h-s of (1.9) is a well defined operator on $D\left(H_{I_{N-1,1}}^{0}\right)$ due to the assumption $D\left(\left(H_{I_{k, i}}^{0}\right)^{\frac{1}{2}}\right) \subseteq$ $D\left(V_{I_{k, i}}\right)$ for any $I_{k, i}$, and the form induced by this operator coincides with $\kappa_{N}(\tau)$ restricted to $D\left(H_{I_{N-1,1}}^{0}\right)$;

2) by [K, Corollary 2.4 , p. 323], the operator $K_{N}$ extends the operator considered in 1). 
The constraint in (1.7) readily implies that

$$
\left\|\left(H_{I_{k, i}}^{0}+1\right)^{-\frac{1}{2}} V_{I_{k, i}}\left(H_{I_{k, i}}^{0}+1\right)^{-\frac{1}{2}}\right\| \leq a .
$$

This motivates introducing the weighted norm

$$
\left\|V_{I_{k, i}}\right\|_{H^{0}}:=\left\|\left(H_{I_{k, i}}^{0}+1\right)^{-\frac{1}{2}} V_{I_{k, i}}\left(H_{I_{k, i}}^{0}+1\right)^{-\frac{1}{2}}\right\| .
$$

Without loss of generality, we may assume that $a=\frac{1}{2}$.

Our results apply to anharmonic quantum crystal models described by Hamiltonians of the form

$$
\left.K_{N}^{\text {crystal }}:=\sum_{j=1}^{N}\left(-\frac{d^{2}}{d x_{j}^{2}}+V\left(x_{j}\right)\right)\right)+t \sum_{j=1}^{N-1} W\left(x_{j}, x_{j+1}\right)=: \sum_{j=1}^{N} H_{j}+t \sum_{j=1}^{N-1} W\left(x_{j}, x_{j+1}\right)
$$

acting on the Hilbert space $\mathcal{H}^{N}:=\otimes_{j=1}^{N} L^{2}\left(\mathbb{R}, d x_{j}\right)$, with $V\left(x_{j}\right) \geq 0, V\left(x_{j}\right) \rightarrow \infty$, for $\left|x_{j}\right| \rightarrow$ $\infty, D\left(\left(H_{j}+H_{j+1}\right)^{\frac{1}{2}}\right) \subseteq D\left(W\left(x_{j}, x_{j+1}\right)\right)$, and $W\left(x_{j}, x_{j+1}\right)$ form-bounded by $H_{j}+H_{j+1}$. The class described above includes the $\phi^{4}$-model on the one-dimensional lattice, corresponding to $V\left(x_{j}\right)=x_{j}^{2}+x_{j}^{4}$ and $W\left(x_{i}, x_{j}\right)=x_{j} x_{j+1}$.

\subsection{Main result}

The main result in this paper is the following theorem proven in Section 3.2; see Theorems 3.6 and 3.8.

Theorem. Under the assumption that (1.4), (1.6) and (1.7) hold, the Hamiltonian $K_{N}$ defined in (1.9) has the following properties: For some $t_{0}>0$ and for any $\tau \in \mathbb{C}$ with $|\tau|<t_{0}$, there exists a suitable invertible operator $U_{N}(\tau)$ such that the operator $U_{N}^{-1}(\tau) K_{N}(\tau) U_{N}(\tau)$ has the following properties:

1. It has a nondegenerate eigenvalue $E_{N}(\tau)$ analytic in $\tau$ for $|\tau|<t_{0}$;

2. the rest of its spectrum is at a distance larger than or equal to $\frac{1}{2}$ from $E_{N}(\tau)$;

3. for $\tau=t \in \mathbb{R}$, the operator $U_{N}(\tau)$ is unitary, and the eigenvalue $E_{N}(\tau \equiv t)$ is the groundstate energy of $K_{N}(t)$.

Our proof is based on the block-diagonalization procedure implemented in [DFPR] for these models but with a real coupling constant. We define

$$
P_{v a c}:=\bigotimes_{i=1}^{N} P_{\Omega_{i}}
$$

(Note that $P_{v a c}$ is the orthogonal projector onto the ground state of the operator $K_{N}(\tau=0)=$ $\sum_{i=1}^{N} H_{i}$.) We construct an invertible operator $U_{N}(\tau)$ acting on $\mathcal{H}^{(N)}$ with the property that, after conjugation, the operator

$$
U_{N}^{-1}(\tau) K_{N}(\tau) U_{N}(\tau)=: \widetilde{K}_{N}(\tau)
$$

is "block-diagonal" with respect to $P_{\text {vac }}, P_{\text {vac }}^{\perp}\left(:=\mathbb{1}-P_{\text {vac }}\right)$

$$
\widetilde{K}_{N}(\tau)=P_{v a c} \widetilde{K}_{N}(\tau) P_{v a c}+P_{v a c}^{\perp} \widetilde{K}_{N}(\tau) P_{v a c}^{\perp}
$$


With $E_{N}(\tau)$ the eigenvalue solving the equation $\widetilde{K}_{N}(\tau) P_{v a c}=E_{N}(\tau) P_{v a c}$, we prove that, for $|\tau| \leq t_{0}$,

$$
\operatorname{spec}\left(P_{\text {vac }}^{\perp}\left(\widetilde{K}_{N}(\tau)-E_{N}(\tau)\right) P_{\text {vac }}^{\perp} \uparrow_{P_{\text {vac }}^{\perp} \mathcal{H}^{(N)}}\right) \cap \overline{\mathbb{D}_{\frac{1}{2}}}=\emptyset,
$$

with $\mathbb{D}_{\rho}:=\{z \in \mathbb{C} ;|z|<\rho\}$, uniformly in $N$.

From the theorem above we also derive the following result (see Proposition 3.9):

Proposition. If the potentials $V_{I_{k, i}}, k \leq \bar{k}$, are invariant under translations, then the limiting function

$$
\varepsilon(\tau):=\lim _{N \rightarrow \infty} \frac{E_{N}(\tau)}{N}
$$

exists for any $|\tau| \leq t_{0}$ and is analytic in $\tau$ for $|\tau|<t_{0}$.

The iterative construction of the operator $U_{N}(\tau)$ coincides with an analogous one for a real coupling constant. For completeness, the scheme of the diagonalization is illustrated in detail in Sect. 2. In Sect. 3, the proof of convergence of our construction of the operator $U_{N}(\tau)$ is discussed by explaining some of the modifications needed in the complex case, in particular the proof of the property displayed in (1.16) is given. Some of the proofs are deferred to Appendix A. The main result of the paper concerning the analyticity of $E_{N}(\tau)$ is presented in Sect. 3.2, Theorem 3.8.

\section{Notation}

1) Notice that $I_{k, q}$ can also be seen as a connected one-dimensional graph with $k$ edges connecting the $k+1$ vertices $q, 1+q, \ldots, k+q$, or as an "interval" of length $k$ whose left end-point coincides with $q$.

2) We use the same symbol for the operator $O_{j}$ acting on $\mathcal{H}_{j}$ and the corresponding operator

$$
\mathbb{1}_{i} \otimes \cdots \otimes \mathbb{1}_{j-1} \otimes O_{j} \otimes \mathbb{1}_{j+1} \cdots \otimes \mathbb{1}_{l}
$$

acting on $\bigotimes_{k=i}^{l} \mathcal{H}_{k}$, for any $i \leq j \leq l$.

3) With the symbol " $\subset$ " we denote strict inclusion, otherwise we use the symbol " $\subseteq$ ".

Acknowledgements. A.P. thanks the Pauli Center, Zürich, for hospitality in Spring 2017 when this project got started. S. D. V. and S. R. are supported by the ERC Advanced Grant 669240 QUEST "Quantum Algebraic Structures and Models". S. D.V., A.P., and S. R. also acknowledge the MIUR Excellence Department Project awarded to the Department of Mathematics, University of Rome Tor Vergata, CUP E83C18000100006.

\section{Local conjugations based on Lie-Schwinger series}

In this section we describe some of the key ideas underlying our proof of the theorem announced in the previous section. We study quantum chains with Hamiltonians $K_{N}(\tau)$ associated with the quadratic form described in (1.9) acting on the Hilbert space $\mathcal{H}^{(N)}$ defined in (1.1). As explained in Sect. 1, our aim is to block-diagonalize $K_{N}(\tau)$, for $|\tau|$ small enough, by conjugating it with a sequence of operators chosen according to the "Lie-Schwinger procedure" (supported on subsets of $\{1, \ldots, N\}$ of successive sites), which for $\tau=t \in \mathbb{R}$ are unitary 
operators. The block-diagonalization will concern operators acting on tensor-product spaces of the type $\mathcal{H}_{q} \otimes \cdots \otimes \mathcal{H}_{k+q}$ (and acting trivially on the remaining tensor factors), and it will be with respect to the projection onto the ground-state ("vacuum") subspace, $\left\{\mathbb{C}\left(\Omega_{q} \otimes \cdots \otimes \Omega_{k+q}\right)\right\}$, contained in $\mathcal{H}_{q} \otimes \cdots \otimes \mathcal{H}_{k+q}$ and its orthogonal complement. Along the way, new interaction terms are created whose supports correspond to increasingly longer intervals (connected subsets) of the chain.

The block-diagonalization procedure for unbounded interactions treated in this paper is formally identical to the scheme introduced in [DFPR]. Hence the formal aspects described in the next section are unchanged w.r.t. [DFPR]. Nevertheless, the lack of self-adjointness of the operators $K_{N}(\tau)$ requires modifications in the proofs, in particular in the control of the spectrum of the operators $G_{I_{k, q}}$; see (2.12).

\subsection{Block-diagonalization: Definitions and formal aspects}

For each $k$, we consider $(N-k)$ block-diagonalization steps, each of them associated with a subset $I_{k, q}, q=1, \ldots, N-k$. The block-diagonalization of the Hamiltonian will be with respect to the subspaces associated with the projectors in (2.4)-(2.5), introduced below. By $(k, q)$ we label the block-diagonalization step associated with $I_{k, q}$. We introduce an ordering amongst these steps:

$$
\left(k^{\prime}, q^{\prime}\right)>(k, q)
$$

if $k^{\prime}>k$ or if $k^{\prime}=k$ and $q^{\prime}>q$.

Our original Hamiltonian is denoted by $K_{N}^{(0, N)}:=K_{N}(\tau)$. We proceed to the first blockdiagonalisation step yielding $K_{N}^{(1,1)}$. The index $(0, N)$ is our initial choice of the index $(k, q)$ : all the on-site terms in the Hamiltonian, i.e, the terms $H_{i}$, are block-diagonal with respect to the subspaces associated with the projectors in (2.4)-(2.5), for $l=0$. Our goal is to arrive at a Hamiltonian of the form

$$
\begin{aligned}
K_{N}^{(k, q):=} & \sum_{i=1}^{N} H_{i}+\tau \sum_{i=1}^{N-1} V_{I_{1, i}}^{(k, q)}+\tau \sum_{i=1}^{N-2} V_{I_{2, i}}^{(k, q)}+\cdots+\tau \sum_{i=1}^{N-k} V_{I_{k, i}}^{(k, q)} \\
& +\tau \sum_{i=1}^{N-k-1} V_{I_{k+1, i}}^{(k, q)}+\cdots+\tau \sum_{i=1}^{2} V_{I_{N-2, i}}^{(k, q)}+\tau V_{I_{N-1,1}}^{(k, q)}
\end{aligned}
$$

(after the block-diagonalization step $(k, q)$ ) with the following properties:

1. For a fixed $I_{l, i}$, the corresponding potential term changes, at each step of the blockdiagonalization procedure, up to the step $(k, q) \equiv(l, i)$; hence $V_{I_{l, i}}^{(k, q)}$ is the potential term associated with the interval $I_{l, i}$ at step $(k, q)$ of the block-diagonalization, and the superscript $(k, q)$ keeps track of the changes in the potential term in step $(k, q)$. The operator $V_{I_{l, i}}^{(k, q)}$ acts as the identity on the spaces $\mathcal{H}_{j}$ for $j \neq i, i+1, \ldots, i+l$; the description of how these terms are created and estimates on their norms are deferred to Sects 2.3 and 3.2;

2. for all sets $I_{l, i}$ with $(l, i) \prec(k, q)$ and for the set $I_{l, i} \equiv I_{k, q}$, the associated potential $V_{I_{l, i}}^{(k, q)}$ is block-diagonal w.r.t. the decomposition of the identity into the sum of projectors

$$
\begin{gathered}
P_{I_{l, i}}^{(-)}:=P_{\Omega_{i}} \otimes P_{\Omega_{i+1}} \otimes \cdots \otimes P_{\Omega_{i+l}}, \\
P_{I_{l, i}}^{(+)}:=\left(P_{\Omega_{i}} \otimes P_{\Omega_{i+1}} \otimes \cdots \otimes P_{\Omega_{i+l}}\right)^{\perp} .
\end{gathered}
$$


Remark 2.1. We warn the reader that new potentials created along the block-diagonalization process are $\tau$-dependent though this is not reflected in our notation.

Remark 2.2. It is important to notice that if $V_{I, i}^{(k, q)}$ is block-diagonal w.r.t. the decomposition of the identity into

$$
P_{I_{l, i}}^{(+)}+P_{I_{l, i}}^{(-)}
$$

i.e.,

$$
V_{I_{l, i}}^{(k, q)}=P_{I_{l, i}}^{(+)} V_{I_{l, i}}^{(k, q)} P_{I_{l, i}}^{(+)}+P_{I_{l, i}}^{(-)} V_{I_{l, i}}^{(k, q)} P_{I_{l, i}}^{(-)},
$$

then, for $I_{l, i} \subset I_{r, j}$, we have that

$$
P_{I_{r, j}}^{(+)}\left[P_{I_{l, i}}^{(+)} V_{I_{l, i}}^{(k, q)} P_{I_{l, i}}^{(+)}+P_{I_{l, i}}^{(-)} V_{I_{l, i}}^{(k, q)} P_{I_{l, i}}^{(-)}\right] P_{I_{r, j}}^{(-)}=0
$$

To see that the first term vanishes, we use that

$$
P_{I_{l, i}}^{(+)} P_{I_{r, j}}^{(-)}=0,
$$

while, in the second term, we use that

$$
P_{I_{l, i}}^{(-)} V_{I_{l, i}}^{(k, q)} P_{I_{l, i}}^{(-)} P_{I_{r, j}}^{(-)}=P_{I_{r, j}}^{(-)} P_{I_{l, i}}^{(-)} V_{I_{l, i}}^{(k, q)} P_{I_{l, i}}^{(-)} P_{I_{r, j}}^{(-)}
$$

and

$$
P_{I_{r, j}}^{(+)} P_{I_{r, j}}^{(-)}=0 .
$$

Hence $V_{I_{l, i}}^{(k, q)}$ is also block-diagonal with respect to the decomposition of the identity into

$$
P_{I_{r, j}}^{(+)}+P_{I_{r, j}}^{(-)}
$$

Note, however, that

$$
P_{I_{r, j}}^{(-)}\left[P_{I_{l, i}}^{(+)} V_{I_{l, i}}^{(k, q)} P_{I_{l, i}}^{(+)}+P_{I_{l, i}}^{(-)} V_{I_{l, i}}^{(k, q)} P_{I_{l, i}}^{(-)}\right] P_{I_{r, j}}^{(-)}=P_{I_{r, j}}^{(-)} V_{I_{l, i}}^{(k, q)} P_{I_{r, j}}^{(-)}
$$

But

$$
P_{I_{r, j}}^{(+)}\left[P_{I_{l, i}}^{(+)} V_{I_{l, i}}^{(k, q)} P_{I_{l, i}}^{(+)}+P_{I_{l, i}}^{(-)} V_{I_{l, i}}^{(k, q)} P_{I_{l, i}}^{(-)}\right] P_{I_{r, j}}^{(+)}
$$

remains as it is.

Remark 2.3. The block-diagonalization procedure that we will implement enjoys the property that the terms block-diagonalized along the process do not change, anymore, in subsequent steps.

\subsection{Lie-Schwinger conjugation associated with $I_{k, q}$}

Here we explain the block-diagonalization procedure from $(k, q-1)$ to $(k, q)$ by which the term $V_{I_{k, q}}^{(k, q-1)}$ is transformed to a new operator, $V_{I_{k, q}}^{(k, q)}$, that is block-diagonal w.r.t. the decomposition of the identity into

$$
P_{I_{k, q}}^{(+)}+P_{I_{k, q}}^{(-)}
$$

We note that, because the first index (i.e., the number of edges of the interval) is changing from $k$ to $k+1$, the steps $(k, N-k) \rightarrow(k+1,1)$ are somewhat different ${ }^{2}$. Here we deal with general

\footnotetext{
${ }^{2}$ The initial step, $(0, N) \rightarrow(1,1)$, is of this type; see the definitions in $(2.23)$ of the terms in the Hamiltonian $K_{N}$ with nearest-neighbor interactions.
} 
steps $(k, q-1) \rightarrow(k, q)$, with $N-k \geq q \geq 2$, and we refer the reader to [DFPR] for the special steps mentioned above that require a slightly different notation.

We recall that the Hamiltonian $K_{N}^{(k, q-1)}$ is given by

$$
\begin{aligned}
K_{N}^{(k, q-1):=} & \sum_{i=1}^{N} H_{i}+\tau \sum_{i=1}^{N-1} V_{I_{1, i}}^{(k, q-1)}+\tau \sum_{i=1}^{N-2} V_{I_{2, i}}^{(k, q-1)}+\cdots+\tau \sum_{i=1}^{N-k} V_{I_{k, i}}^{(k, q-1)} \\
& +\tau \sum_{i=1}^{N-k-1} V_{I_{k+1, i}}^{(k, q-1)}+\cdots+\tau \sum_{i=1}^{2} V_{I_{N-2, i}}^{(k, q-1)}+\tau V_{I_{N-1,1}}^{(k, q-1)}
\end{aligned}
$$

and has the following properties

1. each operator $V_{I_{l, i}}^{(k, q-1)}$ acts as the identity on the spaces $\mathcal{H}_{j}$ for $j \neq i, i+1, \ldots, i+l$. In Sect. 2.3 we explain how these terms are created, and in Sect. 3.2 how their norms can be estimated;

2. each operator $V_{I_{l, i}}^{(k, q-1)}$, with $l<k$ or $l=k$ and $q-1 \geq i$, is block-diagonal w.r.t. the decomposition of the identity into the sum of projectors in (2.4)-(2.5).

Remark 2.4. The term step is used throughout the paper with two slightly different meanings:

i) as level in the block-diagonalization iteration, e.g., $K_{N}^{(k, q)}$ is the Hamiltonian in step $(k, q)$;

ii) for the block-diagonalization procedure to switch from level $(k, q-1)$ to level $(k, q)$, e.g., the step $(k, q-1) \rightarrow(k, q)$.

With the next block-diagonalization step, labeled by $(k, q)$, we want to block-diagonalize the interaction term $V_{I_{k, q}}^{(k, q-1)}$, considering the operator

$$
G_{I_{k, q}}:=\sum_{i \subset I_{k, q}} H_{i}+\tau \sum_{I_{1, i} \subset I_{k, q}} V_{I_{1, i}}^{(k, q-1)}+\cdots+\tau \sum_{I_{k-1, i} \subset I_{k, q}} V_{I_{k-1, i}}^{(k, q-1)}
$$

as the "unperturbed" Hamiltonian. This operator is block-diagonal w.r.t. the decomposition of the identity, i.e.,

$$
G_{I_{k, q}}=P_{I_{k, q}}^{(+)} G_{I_{k, q}} P_{I_{k, q}}^{(+)}+P_{I_{k, q}}^{(-)} G_{I_{k, q}} P_{I_{k, q}}^{(-)}
$$

see Remarks 2.2 and 2.3. We also define

$$
E_{I_{k, q}}:=\left\langle\Omega_{q} \otimes \Omega_{2} \otimes \cdots \otimes \Omega_{k+q}, G_{I_{k, q}} \Omega_{q} \otimes \Omega_{2} \otimes \cdots \otimes \Omega_{k+q}\right\rangle
$$

so that

$$
G_{I_{k, q}} P_{I_{k, q}}^{(-)}=E_{I_{k, q}} P_{I_{k, q}}^{(-)} .
$$

Next, we sketch a convenient formalism used to construct our block-diagonalisation operations; for further details the reader is referred to Sects. 2 and 3 of [DFFR]. For operators $A$ and $B$, we define

$$
\operatorname{ad} A(B):=[A, B],
$$

and, for $n \geq 2$,

$$
a d^{n} A(B):=\left[A, a d^{n-1} A(B)\right] .
$$

This definition is in general only formal for unbounded operators; in fact, the $B$ operators in the formulae below are unbounded. But, as shown in the proof of Theorem 3.4, the formula 
is still meaningful for the operators considered in this paper. In the block-diagonalization step $(k, q)$, we use the operator

$$
S_{I_{k, q}}:=\sum_{j=1}^{\infty} \tau^{j}\left(S_{I_{k, q}}\right)_{j},
$$

where the terms $\left(S_{I_{k, q}}\right)_{j}$ are defined iteratively; (notice that our definition is meaningful, since $\left(V_{I_{k, q}}^{(k, q-1)}\right)_{j}$ depends on the operators $\left(V_{I_{k, q}}^{(k, q-1)}\right)_{1}$ and $\left(S_{I_{k, q}}\right)_{r}$, with $\left.r<j\right)$ :

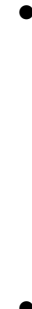

$$
\begin{aligned}
\left(S_{I_{k, q}}\right)_{j}:= & \frac{1}{G_{I_{k, q}}-E_{I_{k, q}}} P_{I_{k, q}}^{(+)}\left(V_{I_{k, q}}^{(k, N-k)}\right)_{j} P_{I_{k, q}}^{(-)} \\
& -P_{I_{k, q}}^{(-)}\left(V_{I_{k, q}}^{(k, N-k)}\right)_{j} P_{I_{k, q}}^{(+)} \frac{1}{G_{I_{k, q}}-E_{I_{k, q}}}
\end{aligned}
$$

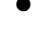

$$
\left(V_{I_{k, q}}^{(k, q-1)}\right)_{1}=V_{I_{k, q}}^{(k, q-1)}
$$

and, for $j \geq 2$,

$\left(V_{I_{k, q}}^{(k, q-1)}\right)_{j}:=$

$$
\begin{aligned}
& =\sum_{p \geq 2, r_{1} \geq 1 \ldots, r_{p} \geq 1 ; r_{1}+\cdots+r_{p}=j} \frac{1}{p !} \operatorname{ad}\left(S_{I_{k, q}}\right)_{r_{1}}\left(\operatorname{ad}\left(S_{I_{k, q}}\right)_{r_{2}} \ldots\left(\operatorname{ad}\left(S_{I_{k, q}}\right)_{r_{p}}\left(G_{I_{k, q}}\right) \ldots\right)\right. \\
& +\sum_{p \geq 1, r_{1} \geq 1 \ldots, r_{p} \geq 1 ; r_{1}+\cdots+r_{p}=j-1} \frac{1}{p !} \operatorname{ad}\left(S_{I_{k, q}}\right)_{r_{1}}\left(\operatorname{ad}\left(S_{I_{k, q}}\right)_{r_{2}} \ldots\left(\operatorname{ad}\left(S_{I_{k, q}}\right)_{r_{p}}\left(V_{I_{k, q}}^{(k, q-1)}\right) \ldots\right) .\right.
\end{aligned}
$$

The operator $S_{I_{k, q}}$ will turn out to be bounded and, consequently, $e^{S_{I_{k, q}}}$ is invertible. We will prove that

$$
K_{N}^{(k, q)}=e^{S_{I_{k, q}}} K_{N}^{(k, q-1)} e^{-S_{I_{k, q}}}
$$

where the 1-h-s in (2.21) involves the effective potentials $V_{l, i}^{(k, q)}$ (see Sect. 2.3) defined in such a way that, a posteriori, the identity above holds.

Remark 2.5. The formal sums defining the operators $\left(V_{I_{k, q}}^{(k, q-1)}\right)_{j}$ and $\left(S_{I_{k, q}}\right)_{j}$, and the series defining $V_{I_{k, q}}^{(k, q)}$ and $S_{I_{k, q}}$, are controlled similarly to the real coupling constant case treated in [DFPR]. This is the content of Sect. 3, with some of the proofs deferred to the Appendix, where the operators $\left(V_{I_{k, q}}^{(k, q-1)}\right)_{j}$ and $V_{I_{k, q}}^{(k, q)}$ will be shown to be bounded in the norm $\|\cdot\|_{H^{0}}$, whereas the operator $S_{I_{k, q}}$ will turn out to be bounded. The more regular behaviour of $S_{I_{k, q}}$ is due to the projectors entering the definition of $\left(S_{I_{k, q}}\right)_{j}$, since one of them, $P_{I_{k, q}}^{(-)}$, is of finite rank.

\subsection{The algorithm $\alpha_{I_{k, q}}$}

The interaction terms arising in our block-diagonalization steps are controlled by an algorithm, $\alpha_{I_{k, q}}$, which determins a map that sends each operator $V_{I_{l, i}}^{(k, q-1)}$ to a corresponding potential term supported on the same interval, but at the next block-diagonalization step, i.e.,

$$
\alpha_{I_{k, q}}\left(V_{I_{l, i}}^{(k, q-1)}\right)=: V_{I_{l, i}}^{(k, q)}
$$




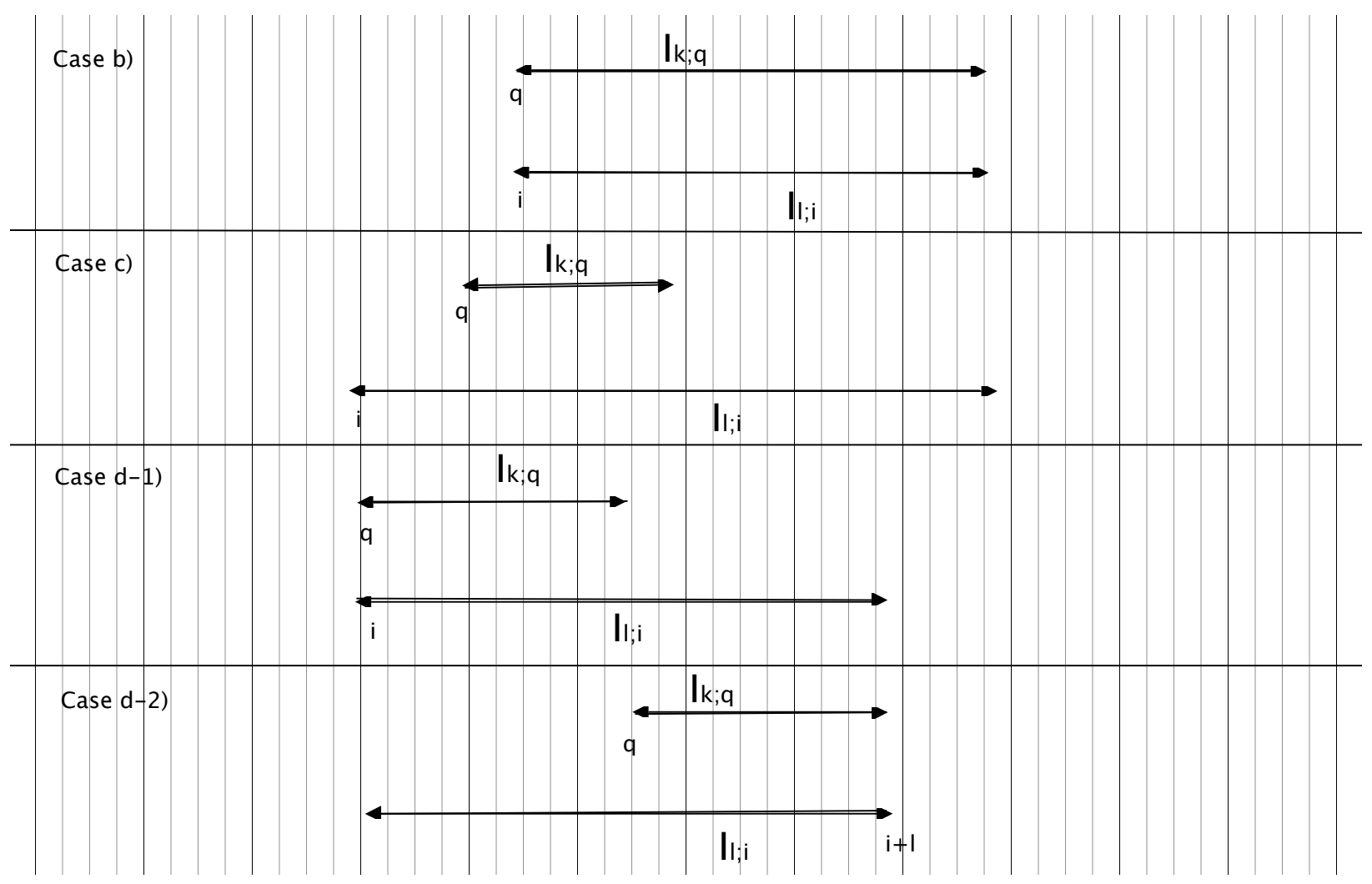

Figure 1: Relative positions of intervals $I_{k, q}$ and $I_{l, i}$

We start from $V_{I_{0, i}}^{(0, N)}:=H_{i}$ and follow the evolution of these operators as well as that of the potential terms. In Definition 2.6, we present the iterative definition of the operators

$$
V_{I_{l, i}}^{(k, q)}:=\alpha_{I_{k, q}}\left(V_{I_{l, i}}^{(k, q-1)}\right)
$$

in terms of the operators, $V_{I_{l, i}}^{(k, q-1)}$, at the previous step $(k, q-1)$, starting from

$$
V_{I_{0, i}}^{(0, N)} \equiv H_{i} \quad, \quad V_{I_{1, i}}^{(0, N)} \equiv V_{I_{1, i}} \quad, \quad V_{I_{l, i}}^{(0, N)}=0 \text { for } l \geq 2 .
$$

We warn the reader that the definitions below involve unbounded operators; see Remark 2.7, below.

Definition 2.6. We assume that, for fixed $(k, q-1)$, with $(k, q-1)>(0, N)$, the operators $V_{I_{l, i}}^{(k, q-1)}$ and $S_{I_{k, q}}$ are well defined, for any $l, i$; or we assume that $(k, q)=(1,1)$ and that the operator $S_{I_{1,1}}$ is well defined. We then define the operators $V_{I_{l, i}}^{(k, q)}$ as follows (but note that if $q=1$ the couple $(k, q-1)$ is replaced by $(k-1, N-k+1)$ in (2.24)-(2.28)); see Fig. 1 for a graphical representation of the different cases b), c) d-1) and d-2, below:

a) in all the following cases

a-i) $l \leq k-1$

a-ii) $I_{l, i} \cap I_{k, q}=\emptyset$;

a-iii) $I_{l, i} \cap I_{k, q} \neq \emptyset$ but $l \geq k$ and $I_{k, q} \nsubseteq I_{l, i}$; 
we define

$$
V_{I_{l, i}}^{(k, q)}:=V_{I_{l, i}}^{(k, q-1)}
$$

b) if $I_{l, i} \equiv I_{k, q}$, we define

$$
V_{I_{l, i}}^{(k, q)}:=\sum_{j=1}^{\infty} \tau^{j-1}\left(V_{I_{l, i}}^{(k, q-1)}\right)_{j}^{\text {diag }}
$$

c) if $I_{k, q} \subset I_{l, i}$ and $i, i+l \notin I_{k, q}$, we define

$$
V_{I_{l, i}}^{(k, q)}:=V_{I_{l, i}}^{(k, q-1)}+\sum_{n=1}^{\infty} \frac{1}{n !} a d^{n} S_{I_{k, i}}\left(V_{I_{l, i}}^{(k, q-1)}\right) ;
$$

d) if $I_{k, q} \subset I_{l, i}$ and either $i$ or $i+l$ belongs to $I_{k, q}$, we define

d-1) if $i$ belongs to $I_{k, q}$, i.e., $q \equiv i$, then

$$
V_{I_{l, i}}^{(k, q)}:=V_{I_{l, i}}^{(k, q-1)}+\sum_{j=0}^{k} \sum_{n=1}^{\infty} \frac{1}{n !} a d^{n} S_{I_{k, i}}\left(V_{I_{l-j, i+j}}^{(k, q-1)}\right) ;
$$

d-2) if $i+l$ belongs to $I_{k, q}$, i.e., $q+k \equiv i+l$ that means $q \equiv i+l-k$, then

$$
V_{I_{l, i}}^{(k, q)}:=V_{I_{l, i}}^{(k, q-1)}+\sum_{j=0}^{k} \sum_{n=1}^{\infty} \frac{1}{n !} a d^{n} S_{I_{k, i+l-k}}\left(V_{I_{l-j, i}}^{(k, q-1)}\right) .
$$

Notice that in both cases, d-1) and d-2), the elements of the sets $\left\{I_{l-j, i+j}\right\}_{j=1}^{k}$ and $\left\{I_{l-j, i}\right\}_{j=1}^{k}$, respectively, are all the intervals, $\mathscr{I}$, such that $\mathscr{I} \cap I_{k, q} \neq \emptyset, \mathscr{I} \nsubseteq I_{k, q}, I_{k, q} \nsubseteq \mathscr{I}$, and $\mathscr{I} \cup I_{k, q} \equiv I_{l, i}$.

Remark 2.7. The results in Theorems 3.4 and the argument in (3.47)-(3.51), below, imply that the quantities defined in (2.24)-(2.28) are to be understood as quadratic forms on the domain $D\left(\left(H_{I_{l i}}^{0}\right)^{\frac{1}{2}}\right)$.

Remark 2.8. Notice that, according to Definition 2.6:

- if $\left(k^{\prime}, q^{\prime}\right)>(l, i)$ then

$$
V_{I_{l, i}}^{\left(k^{\prime}, q^{\prime}\right)}=V_{I_{l, i}}^{(l, i)},
$$

since cases b), c), d-1), and d-2) do not arise;

- for $k \geq 1$ and all allowed choices of $q$,

$$
V_{I_{0, i}}^{(k, q)}=H_{i}
$$

due to a-i);

- the following identity holds

$$
e^{S_{I_{k, q}}}\left(G_{I_{k, q}}+\tau V_{I_{k, q}}^{(k, q-1)}\right) e^{-S_{I_{k, q}}}=G_{I_{k, q}}+\tau \sum_{j=1}^{\infty} \tau^{j-1}\left(V_{I_{k, q}}^{(k, q-1)}\right)_{j}^{d i a g} .
$$

Hence the net result of the conjugation of the sum of the operators $V_{I_{l, i}}^{(k, q-1)}$ appearing on the left side of (2.31) can be re-interpreted as follows: 
a) The operators $V_{I_{l, i}}^{(k, q-1)}$, with $I_{l, i} \subset I_{k, q}$, are kept fixed in the step $(k, q-1) \rightarrow(k, q)$, i.e., we define $V_{I_{l, i}}^{(k, q)}:=V_{I_{l, i}}^{(k, q-1)}$, hence

$$
\begin{aligned}
G_{I_{k, q}} & =\sum_{i \subset I_{k, q}} H_{i}+\tau \sum_{I_{1, i} \subset I_{k, q}} V_{I_{1, i}}^{(k, q-1)}+\cdots+\tau \sum_{I_{k-1, i} \subset I_{k, q}} V_{I_{k-1, i}}^{(k, q-1)} \\
& =\sum_{i \subset I_{k, q}} H_{i}+\tau \sum_{I_{1, i} \subset I_{k, q}} V_{I_{1, i}}^{(k, q)}+\cdots+\tau \sum_{I_{k-1, i} \subset I_{k, q}} V_{I_{k-1, i}}^{(k, q)}
\end{aligned}
$$

b) the operator $V_{I_{k, q}}^{(k, q-1)}$ is transformed to the operator

$$
V_{I_{k, q}}^{(k, q)}:=\sum_{j=1}^{\infty} \tau^{j-1}\left(V_{I_{k, q}}^{(k, q-1)}\right)_{j}^{\text {diag }},
$$

which is block-diagonal, and

$$
\left\|V_{I_{k, q}}^{(k, q)}\right\|_{H^{0}} \leq 2\left\|V_{I_{k, q}}^{(k, q-1)}\right\|_{H^{0}}
$$

as will be shown, assuming that $|\tau|>0$ is sufficiently small;

- the expansion of $e^{S_{I_{k, i}}} V_{I_{l, i}}^{(k, q-1)} e^{-S_{I_{k, i}}}$ in cases c), d-1), and d-2) is controlled (see Theorem 3.4) by exploiting the boundedness of $S_{I_{k, i}}$ and the bound on the weighted operator norm $\left\|V_{I_{l, i}}^{(k, q-1)}\right\|_{H^{0}}$, which are proved iteratively by combining Lemma A.2 with Theorem 3.4. 


\section{Block-diagonalization of $K_{N}(\tau)$ and analiticity of $E_{N}(\tau)$}

In this section we add mathematical rigour to the block-diagonalization procedure described in Sect. 2, and we prove the main result of the paper concerning the analyticity of $E_{N}(\tau)$. The section is divided into three parts. In Sect. 3.1 we study the modifications that are needed (due to the complex coupling constant) to show that $G_{I_{k, q}}$ does not have spectrum in a certain punctured small disk centred at $E_{I_{k, q}}$; in Sect. 3.2 we outline the control of the weighted norm of the effective potentials, (the proofs are deferred to the appendix); in Sect. 3.3 we state and prove our main result, namely Theorem 3.8.

\subsection{Block-diagonalization: Spectrum of the local Hamiltonian $G_{I_{k, q}}$ around $E_{I_{k, q}}$}

To simplify our presentation, we consider a nearest-neighbor interaction with

$$
\left\|V_{I_{1, i}}\right\|_{H^{0}}:=\left\|\left(H_{I_{1, i}}^{0}+1\right)^{-\frac{1}{2}} V_{I_{1, i}}\left(H_{I_{1, i}}^{0}+1\right)^{-\frac{1}{2}}\right\|=\frac{1}{2} .
$$

However, with obvious modifications, our proof can be adapted to general Hamiltonians of the type as in (1.5). Furthermore, we define

$$
\begin{aligned}
& \left\langle V_{I_{j, i}}^{(k, q-1)}\right\rangle \\
:= & \left\langle\Omega_{i} \otimes \Omega_{i+1} \cdots \otimes \Omega_{i+j}, V_{I_{j, i}}^{(k, q-1)} \Omega_{i} \otimes \Omega_{i+1} \cdots \otimes \Omega_{i+j}\right\rangle \\
= & \left\langle\Omega_{i} \otimes \Omega_{i+1} \cdots \otimes \Omega_{i+j},\left(H_{I_{j, i}}^{0}+1\right)^{-\frac{1}{2}} V_{I_{j, i}}^{(k, q-1)}\left(H_{I_{j, i}}^{0}+1\right)^{-\frac{1}{2}} \Omega_{i} \otimes \Omega_{i+1} \cdots \otimes \Omega_{i+j}\right\rangle,
\end{aligned}
$$

which, using the definition in (2.14), implies the following identity

$$
E_{I_{k, q}}=\tau\left\{\sum_{I_{1, i} \subset I_{k, q}}\left\langle V_{I_{1, i}}^{(k, q-1)}\right\rangle+\cdots+\sum_{I_{k-1, i} \subset I_{k, q}}\left\langle V_{I_{k-1, i}}^{(k, q-1)}\right\rangle\right\} .
$$

Our induction hypothesis is that, for $|\tau|>0$ small enough, and for arbitrary $(l, i)$,

$$
\left\|\left(H_{I_{l, i}}^{0}+1\right)^{-\frac{1}{2}} V_{I_{l, i}}^{(k, q-1)}\left(H_{I_{l, i}}^{0}+1\right)^{-\frac{1}{2}}\right\|=:\left\|V_{I_{l, i}}^{(k, q-1)}\right\|_{H^{0}} \leq|\tau|^{\frac{l-1}{4}} .
$$

Remark 3.1. (Domain of $G_{I_{k, q}}$ ) Assuming the bound in (3.5), the formal expression $G_{I_{k, q}}$ is a well-defined closed quadratic form, which we denote by $g_{I_{k, q}}$, on the domain $D\left(\left(H_{I_{k, q}}^{0}\right)^{\frac{1}{2}}\right)$. Hence, as in the definition of the operator associated to the closed quadratic form in (1.9), we can state that, for $|\tau|$ sufficiently small but independent of $k, q$, and $N$ :

i) there is a domain that we call $D\left(G_{I_{k, q}}\right) \subset Q\left(g_{I_{k, q}}\right)$ where an m-sectorial - and thus closed - operator $G_{I_{k, q}} \equiv G_{I_{k, q}}(\tau)$ is defined and the associated form coincides with $g_{I_{k, q}}$ (the operator $G_{I_{k, q}}$ is uniquely determined by the properties in i));

ii) the form domain $Q\left(G_{I_{k, q}}\right)$ coincides with the form domain, $Q\left(H_{I_{k, q}}^{0}\right) \equiv D\left(\left(H_{I_{k, q}}^{0}\right)^{\frac{1}{2}}\right)$, of $H_{I_{k, q}}^{0}$.

We refer the reader to Theorems 3.9 and 2.1 of $[\mathrm{K}]$.

According to the scheme described in Sect. 2.2, the operators $V_{I_{j, i}}^{(k, q-1)}$ are block-diagonalized, for arbitrary $1 \leq j \leq k-1$; i.e.,

$$
V_{I_{j, i}}^{(k, q-1)}=P_{I_{1, i}}^{(+)} V_{I_{j, i}}^{(k, q-1)} P_{I_{1, i}}^{(+)}+P_{I_{1, i}}^{(-)} V_{I_{j, i}}^{(k, q-1)} P_{I_{j, i}}^{(-)}
$$


Hence we can write

$$
\begin{aligned}
P_{I_{k, q}}^{(+)}[ & \left.\sum_{i \subset I_{k ; q}} H_{i}+\tau \sum_{I_{1, i} \subset I_{k, q}} V_{I_{1, i}}^{(k, q-1)}+\cdots++\tau \sum_{I_{k-1, i} \subset I_{k, q}} V_{I_{k-1, i}}^{(k, q-1)}\right] P_{I_{k, q}}^{(+)} \\
=P_{I_{k, q}}^{(+)}[ & \sum_{i \subset I_{k, q}} H_{i} \\
& +\tau \sum_{I_{1, i} \subset I_{k, q}} P_{I_{1, i}}^{(+)} V_{I_{1, i}}^{(k, q-1)} P_{I_{1, i}}^{(+)}+\tau \sum_{I_{1, i} \subset I_{k, q}} P_{I_{1, i}}^{(-)} V_{I_{1, i}}^{(k, q-1)} P_{I_{1, i}}^{(-)}+ \\
& \quad \ldots \\
& \left.+\tau \sum_{I_{k-1, i} \subset I_{k, q}} P_{I_{k-1, i}}^{(+)} V_{I_{k-1, i}}^{(k, q-1)} P_{I_{1, i}}^{(+)}+\tau \sum_{I_{k-1, i} \subset I_{k, q}} P_{I_{k-1, i}}^{(-)} V_{I_{k-1, i}}^{(k, q-1)} P_{I_{k-1, i}}^{(-)}\right] P_{I_{k, q}}^{(+)}
\end{aligned}
$$

Recalling that $P_{I_{j, i}}^{(-)}=\mathbb{1}-P_{I_{j, i}}^{(+)}$, we observe that

$$
P_{I_{j, i}}^{(-)} V_{I_{j, i}}^{(k, q-1)} P_{I_{j, i}}^{(-)}=\left\langle V_{I_{j, i}}^{(k, q-1)}\right\rangle P_{I_{j, i}}^{(-)}=\left\langle V_{I_{j, i}}^{(k, q-1)}\right\rangle-\left\langle V_{I_{j, i}}^{(k, q-1)}\right\rangle P_{I_{j, i}}^{(+)},
$$

and, from (3.3),

$$
\left|\left\langle V_{I_{j, i}}^{(k, q-1)}\right\rangle\right| \leq\left\|\left(H_{I_{j, i}}^{0}+1\right)^{-\frac{1}{2}} V_{I_{j, i}}^{(k, q-1)}\left(H_{I_{j, i}}^{0}+1\right)^{-\frac{1}{2}}\right\| .
$$

Next, we define

$$
\begin{aligned}
\tau \mathcal{V}_{I_{k, q}}:= & \tau \sum_{I_{1, i} \in I_{k, q}} P_{I_{1, i}}^{(+)}\left(V_{I_{1, i}}^{(k, q-1)}-\left\langle V_{I_{1, i}}^{(k, q-1)}\right\rangle\right) P_{I_{1, i}}^{(+)}+ \\
& +\ldots \\
& +\tau \sum_{I_{k-1, i} \in I_{k, q}} P_{I_{k-1, i}}^{(+)}\left(V_{I_{k-1, i}}^{(k, q-1)}-\left\langle V_{I_{k-1, i}}^{(k, q-1)}\right\rangle\right) P_{I_{k-1, i}}^{(+)}
\end{aligned}
$$

Consequently, we have that

$$
\begin{aligned}
& P_{I_{k, q}}^{(+)}\left(G_{I_{k, q}}-E_{I_{k, q}}\right) P_{I_{k, q}}^{(+)} \\
&=P_{I_{k, q}}^{(+)} {\left[\sum_{i \subset I_{k, q}} H_{i}\right.} \\
&+\tau \sum_{I_{1, i} \subset I_{k, q}} P_{I_{1, i}}^{(+)}\left(V_{I_{1, i}}^{(k, q-1)}-\left\langle V_{I_{1, i}}^{(k, q-1)}\right\rangle\right) P_{I_{1, i}}^{(+)}+ \\
&\left.\quad \ldots \quad \sum_{I_{k-1, i} \subset I_{k, q}} P_{I_{k-1, i}}^{(+)}\left(V_{I_{k-1, i}}^{(k, q-1)}-\left\langle V_{I_{k-1, i}}^{(k, q-1)}\right\rangle\right) P_{I_{k-1, i}}^{(+)}\right] P_{I_{k, q}}^{(+)} . \\
&+\tau \\
&=P_{I_{k, q}}^{(+)}\left[\sum_{i \subset I_{k, q}} H_{i}+\tau \mathcal{V}_{I_{k, q}}\right] P_{I_{k, q}}^{(+)},
\end{aligned}
$$

which is a closed operator on $P_{I_{k, q}}^{(+)} D\left(G_{I_{k, q}}\right)$.

Lemma 3.2. Assuming condition (3.5), and choosing $|\tau|$ so small that

$$
1-8 \tau \sum_{j=1}^{+\infty}(j+1)|\tau|^{\frac{j-1}{4}}>0,
$$


the following inequality holds true

$$
\left\|\frac{1}{P_{I_{k, q}}^{(+)}\left(G_{I_{k, q}}-E_{I_{k, q}}-z\right) P_{I_{k, q}}^{(+)}} P_{I_{k, q}}^{(+)}\right\| \leq \frac{2}{1-8|\tau| \sum_{j=1}^{+\infty}(j+1)|\tau|^{\frac{j-1}{4}}} .
$$

for arbitrary $z$ with $|z| \leq \frac{1}{2}$.

Proof.

We propose to use the following expansion, for $|\tau|$ sufficiently small and $z \in \overline{\mathbb{D}_{\frac{1}{2}}}$ :

$$
\begin{aligned}
& \frac{1}{P_{I_{k, q}}^{(+)}\left(G_{I_{k, q}}-E_{I_{k, q}}-z\right) P_{I_{k, q}}^{(+)}} P_{I_{k, q}}^{(+)} \\
= & \left(\frac{1}{P_{I_{k, q}}^{(+)}\left(\sum_{i \subset I_{k, q}} H_{i}-z\right) P_{I_{k, q}}^{(+)}}\right)^{\frac{1}{2}} \times \\
& \times \sum_{l=0}^{\infty}\left\{\left(\frac{1}{P_{I_{k, q}}^{(+)}\left(\sum_{i \subset I_{k, q}} H_{i}-z\right) P_{I_{k, q}}^{(+)}}\right)^{\frac{1}{2}}\left[P_{I_{k, q}}^{(+)} \tau \mathcal{V}_{I_{k, q}} P_{I_{k, q}}^{(+)}\right]\left(\frac{1}{P_{I_{k, q}}^{(+)}\left(\sum_{i \subset I_{k, q}} H_{i}-z\right) P_{I_{k, q}}^{(+)}}\right)^{\frac{1}{2}}\right\}^{l} \\
& \times\left(\frac{1}{P_{I_{k, q}}^{(+)}\left(\sum_{i \subset I_{k, q}} H_{i}-z\right) P_{I_{k, q}}^{(+)}}\right)^{\frac{1}{2}} .
\end{aligned}
$$

To justify this, we need some ingredients. First, we make use of the spectral theorem (recall that $H_{I_{r, i}}^{0}$ is self-adjoint) and the assumption in (1.4) to derive the bound

$$
\left\|P_{I_{r, i}}^{(+)}\left(\frac{H_{I_{r, i}}^{0}+1}{H_{I_{r, i}}^{0}}\right)^{\frac{1}{2}} P_{I_{r, i}}^{(+)}\right\| \leq \sqrt{2} .
$$

By combining (3.5) and (3.13), we then find that

$$
\left\|\left(H_{I_{j, i}}^{0}+1\right)^{-\frac{1}{2}}\left(V_{I_{j, i}}^{(k, q-1)}-\left\langle V_{I_{j, i}}^{(k, q-1)}\right\rangle\right)\left(H_{I_{j, i}}^{0}+1\right)^{-\frac{1}{2}}\right\| \leq 2|\tau|^{\frac{j-1}{4}} .
$$

Next, for each $1 \leq j \leq k-1$, we can make use of the following inequality

$$
\begin{aligned}
& \left\|\left(\frac{1}{P_{I_{k, q}}^{(+)}\left(\sum_{i \subset I_{k, q}} H_{i}-z\right) P_{I_{k, q}}^{(+)}}\right)^{\frac{1}{2}} P_{I_{k, q}}^{(+)}\left[P_{I_{j, i}}^{(+)}\left(V_{I_{j, i}}^{(k, q-1)}-\left\langle V_{I_{j, i}}^{(k, q-1)}\right\rangle\right) P_{I_{j, i}}^{(+)}\right] P_{I_{k, q}}^{(+)}\left(\frac{1}{P_{I_{k, q}}^{(+)}\left(\sum_{i \subset I_{k, q}} H_{i}-z\right) P_{I_{k, q}}^{(+)}}\right)^{\frac{1}{2}}\right\| \\
& \leq\left\|\left(\frac{1}{P_{I_{k, q}}^{(+)}\left(\sum_{i \subset I_{k, q}} H_{i}-z\right) P_{I_{k, q}}^{(+)}}\right)^{\frac{1}{2}} P_{I_{k, q}}^{(+)} P_{I_{j, i}}^{(+)}\left(H_{I_{j, i}}^{0}\right)^{\frac{1}{2}}\left(\frac{H_{I_{j, i}}^{0}+1}{H_{I_{j, i}}^{0}}\right)^{\frac{1}{2}}\right\|^{2} \times \\
& \times\left\|\left(H_{I_{j, i}}^{0}+1\right)^{-\frac{1}{2}}\left(V_{I_{j, i}}^{(k, q-1)}-\left\langle V_{I_{j, i}}^{(k, q-1)}\right\rangle\right)\left(H_{I_{j, i}}^{0}+1\right)^{-\frac{1}{2}}\right\| \\
& \leq\left\|\left(\frac{1}{P_{I_{k, q}}^{(+)}\left(\sum_{i \subset I_{k, q}} H_{i}-z\right) P_{I_{k, q}}^{(+)}}\right)^{\frac{1}{2}} P_{I_{k, q}}^{(+)}\left(H_{I_{j, i}}^{0}\right)^{\frac{1}{2}}\right\|^{2}\left\|P_{I_{j, i}}^{(+)}\left(\frac{H_{I_{j, i}}^{0}+1}{H_{I_{j, i}}^{0}}\right)^{\frac{1}{2}}\right\|^{2} \times \\
& \times\left\|\left(H_{I_{j, i}}^{0}+1\right)^{-\frac{1}{2}}\left(V_{I_{j, i}}^{(k, q-1)}-\left\langle V_{I_{j, i}}^{(k, q-1)}\right\rangle\right)\left(H_{I_{j, i}}^{0}+1\right)^{-\frac{1}{2}}\right\| \\
& =\left\|\left(\frac{1}{P_{I_{k, q}}^{(+)}\left(\sum_{i \subset I_{k, q}} H_{i}-z\right) P_{I_{k, q}}^{(+)}}\right)^{\frac{1}{2}} P_{I_{k, q}}^{(+)} H_{I_{j, i}}^{0} P_{I_{k, q}}^{(+)}\left(\frac{1}{P_{I_{k, q}}^{(+)}\left(\sum_{i \subset I_{k, q}} H_{i}-z\right) P_{I_{k, q}}^{(+)}}\right)^{\frac{1}{2}}\right\| \times \\
& \times\left\|P_{I_{j, i}}^{(+)}\left(\frac{H_{I_{j, i}}^{0}+1}{H_{I_{j, i}}^{0}}\right)^{\frac{1}{2}}\right\|^{2}\left\|\left(H_{I_{j, i}}^{0}+1\right)^{-\frac{1}{2}}\left(V_{I_{j, i}}^{(k, q-1)}-\left\langle V_{I_{j, i}}^{(k, q-1)}\right\rangle\right)\left(H_{I_{j, i}}^{0}+1\right)^{-\frac{1}{2}}\right\| .
\end{aligned}
$$


Recalling (3.28) and (3.29), we finally derive the bound

$$
\begin{gathered}
\quad\left\|\left(\frac{1}{P_{I_{k, q}}^{(+)}\left(\sum_{i \subset I_{k, q}} H_{i}-z\right) P_{I_{k, q}}^{(+)}}\right)^{\frac{1}{2}} P_{I_{k, q}}^{(+)}\left[P_{I_{j, i}}^{(+)}\left(V_{I_{j, i}}^{(k, q-1)}-\left\langle V_{I_{j, i}}^{(k, q-1)}\right\rangle\right) P_{I_{j, i}}^{(+)}\right] P_{I_{k, q}}^{(+)}\left(\frac{1}{P_{I_{k, q}}^{(+)}\left(\sum_{i \subset I_{k, q}} H_{i}-z\right) P_{I_{k, q}}^{(+)}}\right)^{\frac{1}{2}}\right\| \\
\leq \\
\quad 4|\tau|^{\frac{j-1}{4}}\left\|\left(\frac{1}{P_{I_{k, q}}^{(+)}\left(\sum_{i \subset I_{k, q}} H_{i}-z\right) P_{I_{k, q}}^{(+)}}\right)^{\frac{1}{2}} P_{I_{k, q}}^{(+)} H_{I_{j, i}}^{0} P_{I_{k, q}}^{(+)}\left(\frac{1}{P_{I_{k, q}}^{(+)}\left(\sum_{i \subset I_{k, q}} H_{i}-z\right) P_{I_{k, q}}^{(+)}}\right)^{\frac{1}{2}}\right\| .
\end{gathered}
$$

We observe that, for $1 \leq l \leq L \leq N-r$,

$$
\sum_{i=l}^{L} H_{I_{r, i}}^{0} \leq(r+1) \sum_{i=l}^{L+r} H_{i}
$$

and, for $|z| \leq \frac{1}{2}$,

$$
\left\|\left(\frac{1}{P_{I_{k, q}}^{(+)}\left(\sum_{i \subset I_{k, q}} H_{i}-z\right) P_{I_{k, q}}^{(+)}}\right)^{\frac{1}{2}} P_{I_{k, q}}^{(+)}\left(\sum_{i \subset I_{k, q}} H_{i}\right) P_{I_{k, q}}^{(+)}\left(\frac{1}{P_{I_{k, q}}^{(+)}\left(\sum_{i \subset I_{k, q}} H_{i}-z\right) P_{I_{k, q}}^{(+)}}\right)^{\frac{1}{2}}\right\| \leq 2,
$$

which follows from the spectral theorem and from the assumption in (1.4). Hence, it readily follows from (3.36) that

$$
\begin{aligned}
& \left\|\sum_{I_{j, i} \subset I_{k, q}}\left(\frac{1}{P_{I_{k, q}}^{(+)}\left(\sum_{i \subset I_{k, q}} H_{i}-z\right) P_{I_{k, q}}^{(+)}}\right)^{\frac{1}{2}} P_{I_{k, q}}^{(+)}\left[P_{I_{j, i}}^{(+)}\left(V_{I_{j, i}}^{(k, q-1)}-\left\langle V_{I_{j, i}}^{(k, q-1)}\right\rangle\right) P_{I_{j, i}}^{(+)}\right] P_{I_{k, q}}^{(+)}\left(\frac{1}{P_{I_{k, q}}^{(+)}\left(\sum_{i \subset I_{k, q}} H_{i}-z\right) P_{I_{k, q}}^{(+)}}\right)^{\frac{1}{2}}\right\| \\
\leq & 4 \sum_{j=1}^{k-1}|\tau|^{\frac{j-1}{4}}\left\|\left(\frac{1}{P_{I_{k, q}}^{(+)}\left(\sum_{i \subset I_{k, q}} H_{i}-z\right) P_{I_{k, q}}^{(+)}}\right)^{\frac{1}{2}} P_{I_{k, q}}^{(+)} \sum_{I_{j, i} \subset I_{k, q}} H_{I_{j, i}}^{0} P_{I_{k, q}}^{(+)}\left(\frac{1}{P_{I_{k, q}}^{(+)}\left(\sum_{i \subset I_{k, q}} H_{i}-z\right) P_{I_{k, q}}^{(+)}}\right)^{\frac{1}{2}}\right\| \\
\leq & 4 \sum_{j=1}^{k-1}|\tau|^{\frac{j-1}{4}}(j+1)\left\|\left(\frac{1}{P_{I_{k, q}}^{(+)}\left(\sum_{i \subset I_{k, q}} H_{i}-z\right) P_{I_{k, q}}^{(+)}}\right)^{\frac{1}{2}} P_{I_{k, q}}^{(+)}\left(\sum_{i \subset I_{k, q}} H_{i}\right) P_{I_{k, q}}^{(+)}\left(\frac{1}{P_{I_{k, q}}^{(+)}\left(\sum_{i \subset I_{k, q}} H_{i}-z\right) P_{I_{k, q}}^{(+)}}\right)^{\frac{1}{2}}\right\| \\
\leq & 8 \sum_{j=1}^{+\infty}(j+1)|\tau|^{\frac{j-1}{4}},
\end{aligned}
$$

where we have used (3.38) in the last step. Finally, we can conclude that, for $|\tau|$ sufficiently small but independent of $k, q$, and $N$,

$$
\begin{aligned}
& \left\|\sum_{l=0}^{\infty}\left\{\left(\frac{1}{P_{I_{k, q}}^{(+)}\left(\sum_{i \subset I_{k, q}} H_{i}-z\right) P_{I_{k, q}}^{(+)}}\right)^{\frac{1}{2}}\left[P_{I_{k, q}}^{(+)} \tau \mathcal{V}_{I_{k, q}} P_{I_{k, q}}^{(+)}\right]\left(\frac{1}{P_{I_{k, q}}^{(+)}\left(\sum_{i \subset I_{k, q}} H_{i}-z\right) P_{I_{k, q}}^{(+)}}\right)^{\frac{1}{2}}\right\}^{l}\right\| \\
\leq & \frac{1}{1-8|\tau| \sum_{j=1}^{+\infty}(j+1)|\tau|^{\frac{j-1}{4}}}<\infty .
\end{aligned}
$$

Lemma 3.2 implies that, under assumption (3.5), $E_{I_{k, q}}$ is an eigenvalue of the Hamiltonian $G_{I_{k, q}}=G_{I_{k, q}}(\tau)$ isolated from the rest of its spectrum by a distance larger than or equal to $\frac{1}{2}$, for $|\tau|$ sufficiently small but independent of $N, k$, and $q$; as stated in the following Corollary.

Corollary 3.3. Assuming (3.5), and choosing $|\tau|$ sufficiently small, but independent of $N, k$, and $q$, the following statement holds: the spectrum of the Hamiltonian $G_{I_{k, q}}$ in the disk of radius $\frac{1}{2}$ 
centred at $E_{I_{k, q}}$ consists of only $E_{I_{k, q}}$, and $E_{I_{k, q}}$ is the eigenvalue of $G_{I_{k, q}}$ corresponding to the "vacuum" eigenvector, $\bigotimes_{j \in I_{k, q}} \Omega_{j}$, in $\mathcal{H}_{I_{k, q}}$, i.e.,

$$
\begin{aligned}
G_{I_{k, q}} P_{I_{k, q}}^{(-)} & =E_{I_{k, q}} P_{I_{k, q}}^{(-)} \\
& =\left[\tau \sum_{I_{1, i} \subset I_{k, q}}\left\langle V_{I_{1, i}}^{(k, q-1)}\right\rangle+\cdots+\tau \sum_{I_{k-1, i} \subset I_{k, q}}\left\langle V_{I_{k-1, i}}^{(k, q-1)}\right\rangle\right] P_{I_{k, q}}^{(-)},
\end{aligned}
$$

and

$$
G_{I_{k, q}} P_{I_{k, q}}^{(+)}=P_{I_{k, q}}^{(+)} G_{I_{k, q}} P_{I_{k, q}}^{(+)}
$$

with $P_{I_{k, q}}^{(+)}\left(G_{I_{k, q}}-E_{I_{k, q}}-z\right) P_{I_{k, q}}^{(+)}$invertible on $P_{I_{k, q}}^{(+)} \mathcal{H}$ for $|z| \leq \frac{1}{2}$.

\subsection{Block-diagonalization: Bound on $\left\|V_{I_{r, i}}^{(k, q)}\right\|_{H^{0}}$ and consistency of the iterative scheme}

Recall that, according to the rules of the algorithm, the weighted norm of the potentials does not change, i.e., $\left\|V_{I_{r, i}^{(k, q-1)}}\right\|_{H^{0}}=\left\|V_{I_{r, i}}^{(k, q)}\right\|_{H^{0}}$, in the step $(k, q-1) \rightarrow(k, q)$, unless $I_{r, i} \cap I_{k, q} \neq \emptyset$; for more details see [DFPR].

In the theorem below we estimate the change of the norm of the potentials in the blockdiagonalization steps, for each $k$, starting from $k=0$. We have to make use of a lower bound on the distance between $E_{I_{k, q}}$ and the rest of the spectrum of the operator $G_{I_{k, q}}$. We will proceed inductively by showing that, for $|\tau|$ sufficiently small but independent of $r, N, k$, and $q$, the operator-norm bound in (3.5), at step $(k, q-1), q \geq 2$ (for $q=1$ see the footnote), yields control over the spectrum of the Hamiltonian $G_{I_{k, q}}$ in a disk centred at $E_{I_{k, q}}$, (see Corollary 3.3), and the latter provides an essential ingredient for the proof of a bound on the weighted operator norms of the potentials, according to (3.5), at the next $\operatorname{step}^{3}(k, q)$.

Theorem 3.4. Assume that $|\tau| \leq t_{0}$, with $t_{0}$ sufficiently small but independent of $k, q$, and $N$ and such that the assumptions of Lemma A.2 are fulfilled. Then the Hamiltonians $G_{I_{k, q}}$ are well-defined closed operators, and

S1) for any interval $I_{r, i}$, with $r \geq 1$, for $(k, q) \prec(r, i+1)$ and for $(k, q)=(r, i+1)$ the operator

$$
\left(H_{I_{r, i}}^{0}+1\right)^{-\frac{1}{2}} V_{I_{r, i}}^{(k, q)}\left(H_{I_{r, i}}^{0}+1\right)^{-\frac{1}{2}}
$$

has a norm bounded by $|\tau|^{\frac{r-1}{4}}$,

S2) $G_{I_{k, q+1}} P_{I_{k, q+1}}^{(+)}$has no spectrum in the disk $\overline{\mathbb{D}_{\frac{1}{2}}}$ centered at $E_{I_{k, q+1}}$ where $G_{I_{k, q}}$ is defined in (2.12) for $k \geq 2$, and $G_{I_{1, q}}:=H_{q}+H_{q+1}$.

Proof.

The proof is identical to Theorem 4.1 in [DFPR], provided $t$ is replaced by $\tau$ or by $|\tau|$, respectively, depending on the context.

In the next theorem we explain how the Hamiltonian $K_{N}^{(k, q)}$ (see (2.2)) is defined in terms of the potentials $V_{I_{l, j}}^{(k, q)}$ (see Definition 2.6) and prove that, as an operator, it coincides with $e^{S_{I_{k, q}}} K_{N}^{(k, q-1)} e^{-S_{I_{k, q}}}$

\footnotetext{
${ }^{3}$ Recall the special steps of type $(k-1, N-k+1) \rightarrow(k, 1)$.
} 
Theorem 3.5. Assume that $|\tau| \leq t_{0}$. For the Hamiltonian $K_{N}^{(k, q)}$ (see (2.2)), with $k \geq 1$ and $q \geq 2$, the following identity

$$
\begin{aligned}
e^{S_{I_{k, q}}} K_{N}^{(k, q-1)} e^{-S_{I_{k, q}}=} & \sum_{i=1}^{N} H_{i}+\tau \sum_{i=1}^{N-1} V_{I_{1, i}}^{(k, q)}+\tau \sum_{i=1}^{N-2} V_{I_{2, i}}^{(k, q)}+\cdots+\tau \sum_{i=1}^{N-k} V_{I_{k, i}}^{(k, q)} \\
& +\tau \sum_{i=1}^{N-k-1} V_{I_{k+1, i}}^{(k, q)}+\cdots+\tau \sum_{i=1}^{2} V_{I_{N-2, i}}^{(k, q)}+\tau V_{I_{N-1,1}}^{(k, q)}
\end{aligned}
$$

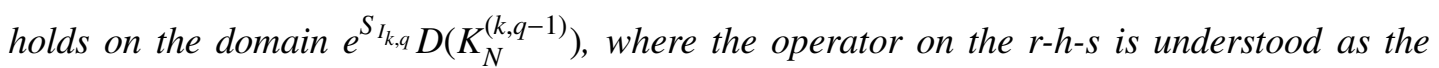
unique $m$-sectorial operator associated with the m-sectorial form

$$
\kappa_{N}^{(k, q)}: D\left(\left(H_{I_{N-1,1}}^{0}\right)^{\frac{1}{2}}\right) \times D\left(\left(H_{I_{N-1,1}}^{0}\right)^{\frac{1}{2}}\right) \rightarrow \mathbb{C}
$$

given by

$$
\begin{aligned}
\kappa_{N}^{(k, q)}(\varphi, \psi):= & \sum_{i=1}^{N}\left\langle H_{i}^{\frac{1}{2}} \varphi, H_{i}^{\frac{1}{2}} \psi\right\rangle+\tau \sum_{i=1}^{N-1}\left\langle V_{I_{1, i}}^{(k, q)} \varphi, \psi\right\rangle+\tau \sum_{i=1}^{N-2}\left\langle V_{I_{2, i}}^{(k, q)} \varphi, \psi\right\rangle+\cdots+\tau \sum_{i=1}^{N-k}\left\langle V_{I_{k, i}}^{(k, q)} \varphi, \psi\right\rangle \\
& +\tau \sum_{i=1}^{N-k-1}\left\langle V_{I_{k+1, i}}^{(k, q)} \varphi, \psi\right\rangle+\cdots+\tau \sum_{i=1}^{2}\left\langle V_{I_{N-2, i}}^{(k, q)} \varphi, \psi\right\rangle+\tau\left\langle V_{I_{N-1,1}}^{(k, q)} \varphi, \psi\right\rangle
\end{aligned}
$$

with $\varphi, \psi \in D\left(\left(H_{I_{N-1,1}}^{0}\right)^{\frac{1}{2}}\right)$.

Proof.

We present the proof for $q \geq 2$, the case $q=1$ can be proved in the same way. We observe that, by following the arguments used in Theorem 4.2 of [DFPR], one can prove that the identity claimed in the statement holds formally. However, our final goal is to prove that (3.46) is in fact an identity between two $m$-sectorial operators (for definitions and results on $m$-sectorial operators the reader is referred to the classic monography by T. Kato $[\mathrm{K}])$.

To this aim, we first observe that $D\left(\left(H_{I_{N-1,1}}^{0}\right)^{\frac{1}{2}}\right)$ is invariant under the action of $e^{S_{I_{k, q}}}$. This is shown by the following estimate.

For any $\varphi \in D\left(\left(H_{I_{N-1,1}}^{0}\right)^{\frac{1}{2}}\right)$ and $m \in \mathbb{N}$ we have

$$
\begin{aligned}
& \left\|\left(H_{I_{r, i}}^{0}\right)^{\frac{1}{2}}\left(S_{I_{k ; q}}\right)^{m} \varphi\right\| \\
= & \left\|\left(H_{I_{r, i}}^{0}\right)^{\frac{1}{2}} \frac{1}{\left(H_{I_{r, i} \backslash I_{k, q}}^{0}+1\right)^{\frac{1}{2}}}\left(H_{I_{r, i} \backslash I_{k, q}}^{0}+1\right)^{\frac{1}{2}}\left(S_{I_{k ; q}}\right)^{m} \varphi\right\| \\
= & \left\|\left(H_{I_{r, i}}^{0}\right)^{\frac{1}{2}} \frac{1}{\left(H_{I_{r, i} \backslash I_{k, q}}^{0}+1\right)^{\frac{1}{2}}}\left(S_{I_{k ; q}}\right)^{m}\left(H_{I_{r, i} \backslash I_{k, q}}^{0}+1\right)^{\frac{1}{2}} \varphi\right\| \\
\leq & \left\|\frac{\left(H_{I_{r, i}}^{0}\right)^{\frac{1}{2}}}{\left(H_{I_{r, i} \backslash I_{k, q}}^{0}+1\right)^{\frac{1}{2}}\left(H_{I_{k, q}}^{0}+1\right)^{\frac{1}{2}}}\right\|\left\|\left(H_{I_{k, q}}^{0}+1\right)^{\frac{1}{2}} S_{I_{k ; q}}\right\|\left\|S_{I_{k, q}}\right\|^{m-1}\left\|\left(H_{I_{r, i} \backslash I_{k, q}}^{0}+1\right)^{\frac{1}{2}} \varphi\right\| \\
\leq & C_{\varphi}^{m},
\end{aligned}
$$

for some constant $C_{\varphi}$ depending on $\varphi$. Here we have exploited estimates (A.4) and (A.5) in Lemma A.2, the spectral theorem for commuting self-adjoint operators, and the assumption $\varphi \in D\left(\left(H_{I_{N-1,1}}^{0}\right)^{\frac{1}{2}}\right)$. 
Next, using the type of manipulations and estimates appearing in the proof of Theorem 3.4, we derive that the relation in (3.46) holds as an identity between matrix elements with vectors in the domain $D\left(\left(H_{I_{N-1,1}}^{0}\right)^{\frac{1}{2}}\right)$, i.e., on the l-h-s of (3.46) we can expand the exponential operator and control the series whenever we consider a matrix element with vectors $\varphi, \psi$ in $D\left(\left(H_{I_{N-1,1}}^{0}\right)^{\frac{1}{2}}\right)$, and then check that they correspond to the analogous matrix elements of the terms on the r-h-s. In this step one has to make sure that also the off-diagonal terms that cancel out on the l-h-s of (3.46) are individually well defined; (this cancellation is indeed the purpose of the conjugation).

Now, thanks to the estimate provided by S1) in Theorem 3.4 and [K, Theorem 3.9, p. 340], the bilinear form $\kappa_{N}^{(k, q)}$ defined in the statement is $m$-sectorial on its domain as a perturbation of a closed symmetric form (the one determined by $H_{I_{N-1,1}}^{0}$ ) by a small perturbation in the sense of quadratic forms, provided $|\tau|$ is chosen small enough, but independent of either $N$ or $(k, q)$. Next, we invoke [K, Theorem 2.1, p. 322] to define $K_{N}^{(k, q)}$ as the unique $m$-sectorial operator associated with the form $\kappa_{N}^{(k, q)}$ whose domain, $D\left(K_{N}^{(k, q)}\right)$, is contained in the domain of the form itself, namely in $D\left(\left(H_{I_{N-1,1}}^{0}\right)^{\frac{1}{2}}\right)$. Using an induction, we see that the operator $e^{S_{I_{k ; q}}} K_{N}^{(k, q-1)} e^{-S_{I_{k ; q}}}$ is $m$-sectorial, since $K_{N}$ is m-sectorial. In light of the invariance property (see (3.47)-(3.51)) proved above, its domain is contained in $D\left(\left(H_{I_{N-1,1}}^{0}\right)^{\frac{1}{2}}\right)$. The identity between matrix elements discussed above implies that the form determined by $e^{S_{I_{k ; q}}} K_{N}^{(k, q-1)} e^{-S_{I_{k ; q}}}$ coincides with the restriction to $e^{S_{I_{k ; q}}} D\left(K_{N}^{(k, q-1)}\right)$ of the form determined by $K_{N}^{(k, q)}$. Hence, a straightforward application of [K, Corollary 2.4, p. 323] shows that the operator $e^{S_{I_{k ; q}}} K_{N}^{(k, q-1)} e^{-S_{I_{k ; q}}}$ is extended by $K_{N}^{(k, q)}$. By recalling that $m$-sectorial operators are $m$-accretive (cf. p. 279-280 in [K]), and no proper inclusions can hold between any two $m$-accretive operators, we conclude that (3.46) is an identity between two $m$-sectorial operators. We recall that $m$-sectorial operators are densely defined; in particular from [K, Corollary 2.4, p. 323] we get that $D\left(H_{I_{N-1,1}^{0}}^{0} \subseteq D\left(K_{N}^{(k, q)}\right)\right.$, using induction, since $K_{N}^{(0, N)} \equiv K_{N}$ and $D\left(H_{I_{N-1,1}}^{0}\right) \subseteq D\left(K_{N}\right)$, as explained in Section 1.1.

Theorem 3.6. Under the assumption that (1.4), (1.6) and (1.7) hold, the complex Hamiltonian $K_{N} \equiv K_{N}(\tau)$ defined in (1.9) has the following properties: There exists some $t_{0}>0$ such that, for any $\tau \in \mathbb{C}$ with $|\tau| \leq t_{0}$, and for all $N<\infty$, an invertible operator $U_{N}(\tau)$ can be constructed such that

1. $U_{N}^{-1}(\tau) K_{N}(\tau) U_{N}(\tau)$ has a nondegenerate eigenvalue, $E_{N}(\tau)$;

2. the rest of its spectrum is at a distance larger than or equal to $\frac{1}{2}$ from $E_{N}(\tau)$;

3. for $\tau \equiv t \in \mathbb{R}$, the eigenvalue $E_{N}(\tau \equiv t)$ is the nondegenerate ground-state energy of $K_{N}(t)$.

Proof. Notice that $K_{N}^{(N-1,1)} \equiv G_{I_{N-1,1}}+\tau V_{I_{N-1,1}}^{(N-1,1)}$. Thus, we have constructed the invertible operator $U_{N}(\tau)$, see (1.14), such that the operator

$$
U_{N}^{-1}(\tau) K_{N}(\tau) U_{N}(\tau)=G_{I_{N-1,1}}+\tau V_{I_{N-1,1}}^{(N-1,1)}=: \widetilde{K}_{N}(\tau)
$$

has the properties in (1.15) and (1.16), which follow from Theorem 3.4 and from (3.24) and (3.44), for $(k, q)=(N-1,1)$, where we also include the block-diagonalized potential $V_{I_{N-1,1}}^{(N-1,1)}$.

Remark 3.7. We stress that, for $\tau$ in the disk of radius $t_{0}$ centered at 0 , all the series used in the construction of all the intermediate Hamiltonians converge uniformly. This result will be invoked in the next theorem wherever those series appear. 


\subsection{Block-diagonalization: Analyticity of $E_{N} \equiv E_{N}(\tau)$}

Theorem 3.8. Under the hypotheses of Theorem 3.6, the eigenvalue $E_{N}(\tau)$ of $U_{N}^{-1}(\tau) K_{N}(\tau) U_{N}(\tau)$ is an analytic function of $\tau$ in $\mathbb{D}_{t_{0}}:=\left\{\tau \in \mathbb{C}:|\tau|<t_{0}\right\}$.

Proof

Since, by construction, $E_{N}(\tau):=\left\langle\widetilde{K}_{N}(\tau) \Omega, \Omega\right\rangle$, with

$$
\widetilde{K}_{N}(\tau):=K_{N}^{(N-1,1)}:=\sum_{i=1}^{N} H_{i}+\tau \sum_{i=1}^{N-1} V_{I_{1, i}}^{(N-1,1)}+\tau \sum_{i=1}^{N-2} V_{I_{2, i}}^{(N-1,1)}+\cdots+\tau V_{I_{N-1,1}}^{(N-1,1)},
$$

it is enough to show that, for all $1 \leq i \leq N-r, 1 \leq r \leq N-1$, the operator-valued functions

$$
\left(\frac{1}{H_{I_{r, i}}^{0}+1}\right)^{\frac{1}{2}} V_{I_{r, i}}^{(N-1,1)}\left(\frac{1}{H_{I_{r, i}}^{0}+1}\right)^{\frac{1}{2}}
$$

are analytic in $\mathbb{D}_{t_{0}}$. Our strategy to show this will consist in establishing the following property:

Property A For any $I_{r, i}$ and $(k, q)$, the (bounded) operators

$$
\left(\frac{1}{H_{I_{r, i}}^{0}+1}\right)^{\frac{1}{2}} V_{I_{r, i}}^{(k, q)}\left(\frac{1}{H_{I_{r, i}}^{0}+1}\right)^{\frac{1}{2}} \equiv\left(\frac{1}{H_{I_{r, i}}^{0}+1}\right)^{\frac{1}{2}} V_{I_{r, i}}^{(k, q)}(\tau)\left(\frac{1}{H_{I_{r, i}}^{0}+1}\right)^{\frac{1}{2}}
$$

are (strongly) analytic in $\tau \in \mathbb{D}_{t_{0}}$.

The result will be achieved by implementing an inductive argument in $(k, q)$. Since we will deal with bounded operators, the analyticity is always understood in the strong sense.

Initial step

At the initial step, $(0, N)$, the only nonzero potentials are $V_{I_{1, j}}^{(0, N)}, 1 \leq j \leq N-1$, and they are $\tau$-independent. Hence the corresponding operators

$$
\left(\frac{1}{H_{I_{1, j}}^{0}+1}\right)^{\frac{1}{2}} V_{I_{1, j}}^{(0, N)}\left(\frac{1}{H_{I_{1, j}}^{0}+1}\right)^{\frac{1}{2}}
$$

are entire operator-valued functions.

Inductive step

We can now move on to the inductive step and show that Property A holds for all $I_{r, i}$ at step $(k, q)$ if it holds for all $\left(k^{\prime}, q^{\prime}\right)<(k, q)$. There are four different cases, a), b), c), and d-1), d-2), as in Definition 2.6. We observe that case a) is trivial. Next, we study case b) in detail.

Case b)

We want to prove that

$$
\left(\frac{1}{H_{I_{k, q}}^{0}+1}\right)^{\frac{1}{2}} V_{I_{k, q}}^{(k, q)}\left(\frac{1}{H_{I_{k, q}}^{0}+1}\right)^{\frac{1}{2}}
$$


is analytic in $\tau \in \mathbb{D}_{t_{0}}$. We recall the formula

$$
V_{k, q}^{(k, q)}=\sum_{j=1}^{\infty} \tau^{j-1}\left(V_{k, q}^{(k, q-1)}\right)_{j}^{d i a g}
$$

where the terms $\left(V_{k, q}^{(k, q-1)}\right)_{j}$ are given by

$$
\begin{aligned}
& \left(V_{I_{k, q}}^{(k, q-1)}\right)_{j}:= \\
& \sum_{p \geq 2, r_{1} \geq 1 \ldots, r_{p} \geq 1 ; r_{1}+\cdots+r_{p}=j} \frac{1}{p !} \operatorname{ad}\left(S_{I_{k, q}}\right)_{r_{1}}\left(\operatorname{ad}\left(S_{I_{k, q}}\right)_{r_{2}} \ldots\left(\operatorname{ad}\left(S_{I_{k, q}}\right)_{r_{p}}\left(G_{I_{k, q}}\right)\right)\right. \\
& +\sum_{p \geq 1, r_{1} \geq 1 \ldots, r_{p} \geq 1 ; r_{1}+\cdots+r_{p}=j-1} \frac{1}{p !} \operatorname{ad}\left(S_{I_{k, q}}\right)_{r_{1}}\left(\operatorname{ad}\left(S_{I_{k, q}}\right)_{r_{2}} \ldots\left(\operatorname{ad}\left(S_{I_{k, q}}\right)_{r_{p}}\left(V_{I_{k, q}}^{(k, q-1)}\right)\right),\right.
\end{aligned}
$$

and the bounded operators $\left(S_{I_{k, q}}\right)_{j}$ are computed by means of the following formula

$$
\left(S_{I_{k, q}}\right)_{j}:=\frac{1}{G_{I_{k, q}}-E_{I_{k, q}}} P_{I_{k, q}}^{(+)}\left(V_{I_{k, q}}^{(k, q-1)}\right)_{j} P_{I_{k, q}}^{(-)}-P_{I_{k, q}}^{(-)}\left(V_{I_{k, q}}^{(k, q-1)}\right)_{j} \frac{1}{G_{I_{k, q}}-E_{I_{k, q}}} P_{I_{k, q}}^{(+)}
$$

Similarly to the control of $\left(V_{I_{k, q}}^{(k, q-1)}\right)_{j}$ we insert the identity operator in the form

$$
\mathbb{1}=\left(\frac{1}{H_{I_{k, q}}^{0}+1}\right)^{\frac{1}{2}}\left(H_{I_{k, q}}^{0}+1\right)^{\frac{1}{2}}=\left(H_{I_{k, q}}^{0}+1\right)^{\frac{1}{2}}\left(\frac{1}{H_{I_{k, q}}^{0}+1}\right)^{\frac{1}{2}}
$$

in a suitable way to express (3.57) in terms of the operators

$$
\left(\frac{1}{H_{I_{k, q}}^{0}+1}\right)^{\frac{1}{2}} V_{I_{k, q}}^{(k, q-1)}\left(\frac{1}{H_{I_{k, q}}^{0}+1}\right)^{\frac{1}{2}}
$$

and

$$
\left(S_{I_{k, q}}\right)_{r}\left(H_{I_{k, q}}^{0}+1\right)^{\frac{1}{2}},\left(H_{I_{k, q}}^{0}+1\right)^{\frac{1}{2}}\left(S_{I_{k, q}}\right)_{r}
$$

with $r<j$. Then, assuming that Property A holds at step $(k, q-1)$, saying that, for all $I_{r, i}$ and for all $\left(k^{\prime}, q^{\prime}\right) \prec(k, q)$, the operators

$$
\left(\frac{1}{H_{I_{r, i}}^{0}+1}\right)^{\frac{1}{2}} V_{I_{r, i}}^{(k, q-1)}\left(\frac{1}{H_{I_{r, i}}^{0}+1}\right)^{\frac{1}{2}}
$$

are analytic in $\mathbb{D}_{t_{0}}$, we can implement an induction on $j$ and prove that, for all $j$, the operators

$$
\left(\frac{1}{H_{I_{k, q}}^{0}+1}\right)^{\frac{1}{2}}\left(V_{I_{k, q}}^{(k, q-1)}\right)_{j}\left(\frac{1}{H_{I_{k, q}}^{0}+1}\right)^{\frac{1}{2}}
$$

are analytic in $\tau \in \mathbb{D}_{t_{0}}$, where the result for $j=1$ is precisely Property A at step $(k, q-1)$. Indeed, suppose it is true for all $j^{\prime}<j$, then, using Property A at step $(k, q-1)$, we get that the operators

$$
\left.\left(S_{I_{k, q}}\right)_{j^{\prime}}\left(H_{I_{k, q}}^{0}+1\right)^{\frac{1}{2}}, H_{I_{k, q}}^{0}+1\right)^{\frac{1}{2}}\left(S_{I_{k, q}}\right)_{j^{\prime}}
$$

are analytic in the same open disk, too, since they are obtained as products of the operators, which are analytic in $\tau$,

$$
\left(\frac{1}{H_{I_{k, q}}^{0}+1}\right)^{\frac{1}{2}}\left(V_{I_{k, q}}^{(k, q-1)}\right)_{j^{\prime}}\left(\frac{1}{H_{I_{k, q}}^{0}+1}\right)^{\frac{1}{2}}
$$


with the operators

$$
\frac{1}{G_{I_{k, q}}-E_{I_{k, q}}} P_{I_{k, q}}^{(+)}\left(H_{I_{k, q}}^{0}+1\right)^{\frac{1}{2}},\left(H_{I_{k, q}}^{0}+1\right)^{\frac{1}{2}} P_{I_{k, q}}^{(+)} \frac{1}{G_{I_{k, q}}-E_{I_{k, q}}}
$$

and the latter are analytic in $\tau$ because of the expansion in (3.25). Indeed:

1) all the summands of the series in (3.25) are analytic in the same disk by Property A at step $(k, q-1)$;

2) the series are norm convergent uniformly for $|\tau| \leq t_{0}$, (see Remark 3.7).

Finally, from the proof of Lemma A.2, the series

$$
\left(\frac{1}{H_{I_{k, q}}^{0}+1}\right)^{\frac{1}{2}} V_{k, q}^{(k, q)}\left(\frac{1}{H_{I_{k, q}}^{0}+1}\right)^{\frac{1}{2}}:=\sum_{j=1}^{\infty} \tau^{j-1}\left(\frac{1}{H_{I_{k, q}}^{0}+1}\right)^{\frac{1}{2}}\left(V_{k, q}^{(k, q-1)}\right)_{j}^{\operatorname{diag}}\left(\frac{1}{H_{I_{k, q}}^{0}+1}\right)^{\frac{1}{2}}
$$

is then easily seen to converge uniformly in $\tau$ for $|\tau| \leq t_{0}$. Thus, we can conclude that the l-h-s of (3.69) is analytic in $\mathbb{D}_{t_{0}}$, too.

Case c)

We start by recalling that, in this case,

$$
\begin{aligned}
& \left(\frac{1}{H_{I_{r, i}}^{0}+1}\right)^{\frac{1}{2}} V_{I_{r, i}}^{(k, q)}\left(\frac{1}{H_{I_{r, i}}^{0}+1}\right)^{\frac{1}{2}} \\
:= & \left(\frac{1}{H_{I_{r, i}}^{0}+1}\right)^{\frac{1}{2}} V_{I_{r, i}}^{(k, q-1)}\left(\frac{1}{H_{I_{r, i}}^{0}+1}\right)^{\frac{1}{2}} \\
& +\left(\frac{1}{H_{I_{r, i}}^{0}+1}\right)^{\frac{1}{2}}\left\{\sum_{n=1}^{\infty} \frac{1}{n !} a d^{n} S_{I_{k, q}}\left(V_{I_{r, i}}^{(k, q-1)}\right)\right\}\left(\frac{1}{\left.H_{I_{r, i}}^{0}+1\right)}\right)^{\frac{1}{2}} .
\end{aligned}
$$

The procedure to be used is quite similar to case b). Just as in controlling the norm in Theorem 3.4 , we insert the identity in the form

$$
\mathbb{1}=\left(H_{I_{r, i}}^{0}+1\right)^{\frac{1}{2}}\left(\frac{1}{H_{I_{r, i}}^{0}+1}\right)^{\frac{1}{2}}, \mathbb{1}=\left(\frac{1}{H_{I_{r, i}}^{0}+1}\right)^{\frac{1}{2}}\left(H_{I_{r, i}}^{0}+1\right)^{\frac{1}{2}}
$$

on the left and the right side of $V_{I_{r, i}}^{(k, q-1)}$, respectively. By combining the arguments used for case b) and the estimates in Lemma A.2 leading to (A.4) and (A.5), we derive that

$$
S_{I_{k, q}} \quad, \quad S_{I_{k, q}}\left(H_{I_{r, i}}^{0}+1\right)^{\frac{1}{2}}, \quad \text { and }\left(H_{I_{r, i}}^{0}+1\right)^{\frac{1}{2}} S_{I_{k, q}}
$$

are analytic in $\tau \in \mathbb{D}_{t_{0}}$. Hence we conclude that the series

$$
\sum_{n=1}^{\infty} \frac{1}{n !} a d^{n} S_{I_{k, q}}\left(V_{I_{r, i}}^{(k, q-1)}\right)
$$

consists of analytic operators, for $\tau$ in $\mathbb{D}_{t_{0}}$, which, according to the proof of Theorem 3.4, converge uniformly, for $|\tau| \leq t_{0}$. Hence, $V_{I_{r, i}}^{(k, q)}$ is analytic in $\mathbb{D}_{t_{0}}$, too.

Cases $d-1), d-2$ )

These two cases are very similar to case c), and we omit the proof. 
As an application of the techniques we have developed in the present work, we show that the limiting function

$$
\lim _{N \rightarrow \infty} \frac{E_{N}(\tau)}{N}
$$

is well defined and analytic in $\tau \in \mathbb{D}_{t_{0}}$ (as in the foregoing result), provided the chain is invariant under translations in a sense specified below. Recall that each $\mathcal{H}_{i}$ is a copy of a Hilbert $\mathcal{H}$, so that, given a vector $\varphi \in \mathcal{H}$, we call $\varphi_{i}$ its representative in $\mathcal{H}_{i}$. For $j \in \mathbb{N}$, we consider the unitary operators

$$
\mathcal{U}_{(j)_{N}}: \mathcal{H}_{i} \rightarrow \mathcal{H}_{(i+j)_{N}} \quad, \quad \mathcal{U}_{(j)_{N}}\left(\varphi_{i}\right):=\varphi_{(i+j)_{N}},
$$

where $(i+j)_{N}:=i+j-k N$ with $k$ the smallest number in $\mathbb{N}_{0} \equiv \mathbb{N} \cup\{0\}$ such that $0 \leq i+j-k N \leq$ $N$. In particular $\mathcal{U}_{(j)_{N}} \Omega_{i}=\Omega_{(i+j)_{N}}$. Define

$$
\mathcal{U}_{(j)_{N}}: \mathcal{H}^{(N)} \rightarrow \mathcal{H}^{(N)}
$$

as

$$
\mathcal{U}_{(j)_{N}}\left(\mathcal{H}^{(N)}\right):=\left(\mathcal{U}_{(j)_{N}} \mathcal{H}_{1}\right) \otimes\left(\mathcal{U}_{(j)_{N}} \mathcal{H}_{2}\right) \otimes \cdots \otimes\left(\mathcal{U}_{(j)_{N}} \mathcal{H}_{N-1}\right) \otimes\left(\mathcal{U}_{(j)_{N}} \mathcal{H}_{N}\right)
$$

Proposition 3.9. Under the hypotheses of Theorem 3.6, and assuming that in (1.9) the potentials $V_{I_{k, i}}, k \leq \bar{k}$, depend only on the length, $k$, of the interval $I_{k, i}$, i.e., for any $0 \leq i \leq N$ and any $j$

$$
V_{I_{k,(i+j)_{N}}}=\mathcal{U}_{(j)_{N}} V_{I_{k, i}} \mathcal{U}_{(j)_{N}}^{*}
$$

the limiting function

$$
\varepsilon(\tau):=\lim _{N \rightarrow \infty} \frac{E_{N}(\tau)}{N}
$$

exists for any $|\tau| \leq t_{0}$ and is analytic in $\tau$ in the open disc $\mathbb{D}_{t_{0}}$.

Proof

Thanks to the local features of the algorithm $\alpha_{I_{k, q}}$, it is not too difficult to realise that the effective potentials $V_{I_{l, i}}^{(k, q)}$ created in the course of our construction enjoy the same covariance property assumed for the potentials $V_{I_{k, i}}$ entering the initial Hamiltonian $K_{N}$, i.e.,

$$
V_{I_{k,(i+j)_{N}}}^{(k, q)}=\mathcal{U}_{(j)_{N}} V_{I_{k, i}}^{(k, q)} \mathcal{U}_{(j)_{N}}^{*}
$$

Consequently, the total energy $E_{N}$ admits a decomposition into a sum of the type

$$
E_{N}(\tau)=\sum_{l=1}^{N-1} n_{l} E_{l}^{(N-1,1)}(\tau),
$$

where $n_{l}=N-l$ is the number of subsequent sets ("intervals") of length $l$ contained in $1,2, \ldots, N$, and $E_{l}^{(N-1,1)}$ is the common expectation value of the effective potentials of length $l$ in the final step, $(N-1,1)$, in the vacuum vector. Notice that by construction $E_{l}^{(N-1,1)}(\tau)$ coincides with the energy, $E_{l}(\tau)$, of the same chain but of length $l$. From Theorem 3.8 we know that this function, $E_{l}(\tau)$, is analytic in $\mathbb{D}_{t_{0}}$. Hence, both existence and analyticity of $\varepsilon(\tau)$ follow if the sequence of functions $\left\{\frac{E_{N}(\tau)}{N}\right\}_{N \in \mathbb{N}}$ is shown to be uniformly Cauchy in the disk $|\tau|<t_{0}$. 
To show this, note that the estimates on the $\|\cdot\|_{H_{0}}$ norms of the effective potentials readily imply that the inequality $\left|E_{l}^{(N-1,1)}\right| \leq 2|\tau|^{\frac{l-1}{4}}$ holds true for any natural number $l$. Next, let $M>N$ be two positive integers. Then, for any $\delta>0$ there is some $N_{\delta}$ such that, for $N>N_{\delta}$, we can estimate

$$
\begin{aligned}
\left|\frac{E_{N}(\tau)}{N}-\frac{E_{M}(\tau)}{M}\right| & \leq \sum_{l=1}^{N}\left|\frac{N-l}{N}-\frac{M-l}{M}\right|\left|E_{l}\right|+\sum_{l=N+1}^{M} \frac{N-l}{M}\left|E_{l}^{(N-1,1)}\right| \\
& \leq \frac{M-N}{N M} \sum_{l=1}^{N} l \cdot\left|E_{l}^{(N-1,1)}\right|+\sum_{l=N+1}^{\infty}\left|E_{l}^{(N-1,1)}\right| \leq \frac{2}{N} \sum_{l=1}^{N} l \cdot|\tau|^{\frac{l-1}{4}}+2 \sum_{l=N+1}^{\infty}|\tau|^{\frac{l-1}{4}} \\
& \leq 2\left(\frac{C}{N}+R_{N}\right) \leq \delta,
\end{aligned}
$$

for any $\tau$ such that $|\tau| \leq t_{0}$, where $C$ is a universal constant and $R_{N}$ depends only on $N$, since the series of functions $\sum_{l=1}^{\infty}|\tau|^{\frac{l-1}{4}}$ and $\sum_{l=1}^{N} l \cdot|\tau|^{\frac{l-1}{4}}$ converge uniformly in $|\tau|$, for $|\tau| \leq t_{0}$, with $t_{0}$ as in Theorem 3.8 . 


\section{A Appendix}

Lemma A.1. Assume that $\left\|V_{I_{r, i}}^{(k, q-1)}\right\|_{H_{0}} \leq|\tau|^{\frac{r-1}{4}}$ and define

$$
\Delta_{I_{k, q}}:=\frac{1-8|\tau| \sum_{j=1}^{\infty}(j+1)|\tau|^{\frac{j-1}{4}}}{2},
$$

then for $|\tau|$ sufficiently small and independent of $k, q$, and $N$,

$$
\left\|\frac{1}{G_{I_{k, q}}-E_{I_{k, q}}} P_{I_{k, q}}^{+}\left(H_{I_{k, q}}^{0}+1\right)^{\frac{1}{2}}\right\| \leq \frac{\sqrt{2}}{\Delta_{I_{k, q}}} .
$$

Proof.

The proof follows from the Neumann expansion in (3.25)-(3.27), from the estimate in (3.42)(3.43), and from the spectral theorem.

Lemma A.2. Assume $\left\|V_{I_{r i}}^{(k, q-1)}\right\|_{H_{0}} \leq|\tau|^{\frac{r-1}{4}}$ and that $|\tau|$ is sufficiently small such that $\Delta_{I_{k, q}} \geq \frac{1}{2}$ (see Corollary 3.3). Then, for arbitrary $N, k \geq 1$, and $q \geq 2$, the inequalities

$$
\begin{aligned}
& \left\|V_{I_{k, q}}^{(k, q)}\right\|_{H_{0}} \leq 2\left\|V_{I_{k, q}^{(k, q-1)}}\right\|_{H_{0}}, \\
& \left\|S_{I_{k, q}}\right\| \leq A|\tau|\left\|V_{I_{k, q}^{(k, q-1)}}\right\|_{H_{0}},
\end{aligned}
$$

and

$$
\left\|S_{I_{k, q}}\left(H_{I_{k, q}}^{0}+1\right)^{\frac{1}{2}}\right\|=\left\|\left(H_{I_{k, q}}^{0}+1\right)^{\frac{1}{2}} S_{I_{k, q}}\right\| \leq B|\tau|\left\|V_{I_{k, q}}^{(k, q-1)}\right\|_{H_{0}}
$$

hold true for universal constants $A$ and B. For $q=1, V_{I_{k, q}}^{(k, q-1)}$ is replaced by $V_{I_{k, q}}^{(k-1, N-k+1)}$ in the right side of (A.3), (A.4), and (A.5).

Proof

In the following we assume $q \geq 2$; if $q=1$ an analogous proof holds. We recall that

$$
V_{I_{k, q}}^{(k, q)}:=\sum_{j=1}^{\infty} \tau^{j-1}\left(V_{I_{k, q}}^{(k, q-1)}\right)_{j}^{\text {diag }}
$$

and

$$
S_{I_{k, q}}:=\sum_{j=1}^{\infty} \tau^{j}\left(S_{I_{k, q}}\right)_{j}
$$

with

$$
\left(V_{I_{k, q}}^{(k, q-1)}\right)_{1}=V_{I_{k, q}}^{(k, q-1)}
$$

and, for $j \geq 2$,

$$
\begin{aligned}
& \left(V_{I_{k, q}}^{(k, q-1)}\right)_{j}:= \\
& \sum_{p \geq 2, r_{1} \geq 1 \ldots, r_{p} \geq 1 ; r_{1}+\cdots+r_{p}=j} \frac{1}{p !} \operatorname{ad}\left(S_{I_{k, q}}\right)_{r_{1}}\left(\operatorname{ad}\left(S_{I_{k, q}}\right)_{r_{2}} \ldots\left(\operatorname{ad}\left(S_{I_{k, q}}\right)_{r_{p}}\left(G_{I_{k, q}}\right)\right)\right. \\
& +\sum_{p \geq 1, r_{1} \geq 1 \ldots, r_{p} \geq 1 ; r_{1}+\cdots+r_{p}=j-1} \frac{1}{p !} \operatorname{ad}\left(S_{I_{k, q}}\right)_{r_{1}}\left(\operatorname{ad}\left(S_{I_{k, q}}\right)_{r_{2}} \ldots\left(\operatorname{ad}\left(S_{I_{k, q}}\right)_{r_{p}}\left(V_{I_{k, q}}^{(k, q-1)}\right)\right),\right.
\end{aligned}
$$


and

$$
\begin{aligned}
\left(S_{I_{k, q}}\right)_{j}:= & \frac{1}{G_{I_{k, q}}-E_{I_{k, q}}} P_{I_{k, q}}^{(+)}\left(V_{I_{k, q}}^{(k, q-1)}\right)_{j} P_{I_{k, q}}^{(-)} \\
& -P_{I_{k, q}}^{(-)}\left(V_{I_{k, q}}^{(k, q-1)}\right)_{j} P_{I_{k, q}}^{(+)} \frac{1}{G_{I_{k, q}}-E_{I_{k, q}}} .
\end{aligned}
$$

where $j \geq 1$.

From the lines above, we have that

$$
\begin{aligned}
\operatorname{ad}\left(S_{I_{k, q}}\right)_{r_{p}}\left(G_{I_{k, q}}\right) & =\operatorname{ad}\left(S_{I_{k, q}}\right)_{r_{p}}\left(G_{I_{k, q}}-E_{I_{k, q}}\right) \\
& =-P_{I_{k, q}}^{(+)}\left(V_{I_{k, q}}^{(k, q-1)}\right)_{r_{p}} P_{I_{k, q}}^{(-)}-P_{I_{k, q}}^{(-)}\left(V_{I_{k, q}}^{(k, q-1)}\right)_{r_{p}} P_{I_{k, q}}^{(+)} .
\end{aligned}
$$

We start by showing the following inequality:

$$
\left\|\left(S_{I_{k, q}}\right)_{j}\right\| \leq \frac{2 \sqrt{2}}{\Delta_{I_{k, q}}}\left\|\left(V_{I_{k, q}}^{(k, q-1)}\right)_{j}\right\|_{H^{0}}
$$

where $\left\|\left(V_{I_{k, q}}^{(k, q-1)}\right)_{j}\right\|_{H_{0}}$ will turn out to be bounded in the next step. Going back to estimate (A.14), the inequality in (A.14) is proven by means of the following computation:

$$
\begin{aligned}
& \left\|\left(S_{I_{k, q}}\right)_{j}\right\| \\
\leq & 2\left\|\frac{1}{G_{I_{k, q}}-E_{I_{k, q}}} P_{I_{k, q}}^{(+)}\left(V_{I_{k, q}}^{(k, q-1)}\right)_{j} P_{I_{k, q}}^{(-)}\right\| \\
= & 2\left\|\frac{1}{G_{I_{k, q}}-E_{I_{k, q}}} P_{I_{k, q}}^{(+)}\left(H_{I_{k, q}}^{0}+1\right)^{\frac{1}{2}}\left(H_{I_{k, q}}^{0}+1\right)^{-\frac{1}{2}}\left(V_{I_{k, q}}^{(k, q-1)}\right)_{j}\left(H_{I_{k, q}}^{0}+1\right)^{-\frac{1}{2}} P_{I_{k, q}}^{(-)}\right\| \\
\leq & 2\left\|\frac{1}{G_{I_{k, q}}-E_{I_{k, q}}} P_{I_{k, q}}^{(+)}\left(H_{I_{k, q}}^{0}+1\right)^{\frac{1}{2}}\right\|\left\|\left(V_{I_{k, q}}^{(k, q-1)}\right)_{j}\right\|_{H^{0}} \\
\leq & \frac{2 \sqrt{2}}{\Delta_{I_{k, q}}}\left\|\left(V_{I_{k, q}}^{(k, q-1)}\right)_{j}\right\|_{H^{0}},
\end{aligned}
$$

where we have used (A.2) for the last inequality.

Analogously, making use of (A.2) and $\left(H_{I_{k, q}}^{0}+1\right)^{\frac{1}{2}} P_{I_{k, q}}^{(-)}=P_{I_{k, q}}^{(-)}$, we estimate

$$
\left\|\left(S_{I_{k, q}}\right)_{j}\left(H_{I_{k, q}}^{0}+1\right)^{\frac{1}{2}}\right\| \leq \frac{2+\sqrt{2}}{\Delta_{I_{k, q}}}\left\|\left(V_{I_{k, q}}^{(k, q-1)}\right)_{j}\right\|_{H^{0}} .
$$

Next, we want to prove that

$$
\begin{aligned}
\left\|\left(V_{I_{k, q}}^{(k, q-1)}\right)_{j}\right\|_{H^{0}} & \leq \\
\sum_{p=2}^{j} \frac{(2 c)^{p}}{p !} & \sum_{r_{1} \geq 1 \ldots, r_{p} \geq 1 ; r_{1}+\cdots+r_{p}=j}\left\|\left(V_{I_{k, q}}^{(k, q-1)}\right)_{r_{1}}\right\|_{H^{0}}\left\|\left(V_{I_{k, q}}^{(k, q-1)}\right)_{r_{2}}\right\|_{H^{0}} \ldots\left\|\left(V_{I_{k, q}}^{(k, q-1)}\right)_{r_{p}}\right\|_{H^{0}} \\
+2\left\|V_{I_{k, q}}^{(k, q-1)}\right\| & \sum_{p=1}^{j-1} \frac{(2 c)^{p}}{p !} \sum_{r_{1} \geq 1 \ldots, r_{p} \geq 1 ; r_{1}+\cdots+r_{p}=j-1}\left\|\left(V_{I_{k, q}}^{(k, q-1)}\right)_{r_{1}}\right\|_{H^{0}}\left\|\left(V_{I_{k, q}}^{(k, q-1)}\right)_{r_{2}}\right\|_{H^{0}} \ldots\left\|\left(V_{I_{k, q}}^{(k, q-1)}\right)_{r_{p}}\right\|_{H^{0}},
\end{aligned}
$$


where $c:=\frac{2+\sqrt{2}}{\Delta_{I_{k, q}}}\left(>\frac{2 \sqrt{2}}{\Delta_{I_{k, q}}}\right)$. In order to show this, we observe that formula (A.8) giving $\left(V_{I_{k, q}}^{(k, q-1)}\right)_{j}$ contains two sums. We first deal with the second sum, namely

$$
\sum_{p \geq 1, r_{1} \geq 1 \ldots, r_{p} \geq 1 ; r_{1}+\cdots+r_{p}=j-1} \frac{1}{p !} \operatorname{ad}\left(S_{I_{k, q}}\right)_{r_{1}}\left(\operatorname{ad}\left(S_{I_{k, q}}\right)_{r_{2}} \ldots\left(\operatorname{ad} .\left(S_{I_{k, q}}\right)_{r_{p}}\left(V_{I_{k, q}}^{(k, q-1)}\right)\right) .\right.
$$

Each summand of the above sum is in turn a sum of $2^{p}$ terms which, up to a sign, are permutations of

$$
\left(S_{I_{k, q}}\right)_{r_{1}}\left(S_{I_{k, q}}\right)_{r_{2}} \ldots\left(S_{I_{k, q}}\right)_{r_{p}} V_{I_{k, q}}^{(k, q-1)}
$$

with the potential $V_{I_{k, q}}^{(k, q-1)}$ which is allowed to appear at any position. It suffices to study only one of these products, for the others can be treated in the same way. For instance, we can treat

$$
\left(S_{I_{k, q}}\right)_{r_{1}} V_{I_{k, q}}^{(k, q-1)}\left(S_{I_{k, q}}\right)_{r_{2}} \ldots\left(S_{I_{k, q}}\right)_{r_{p}}
$$

Notice that

$$
\begin{aligned}
& \left\|\left(S_{I_{k, q}}\right)_{r_{1}} V_{I_{k, q}}^{(k, q-1)}\left(S_{I_{k, q}}\right)_{r_{2}} \ldots\left(S_{I_{k, q}}\right)_{r_{p}}\right\|_{H^{0}} \\
= & \left\|\left(H_{I_{k, q}}^{0}+1\right)^{-\frac{1}{2}}\left(S_{I_{k, q}}\right)_{r_{1}}\left(H_{I_{k, q}}^{0}+1\right)^{\frac{1}{2}}\left(H_{I_{k, q}}^{0}+1\right)^{-\frac{1}{2}} V_{I_{k, q}}^{(k, q-1)}\left(H_{I_{k, q}}^{0}+1\right)^{-\frac{1}{2}}\left(H_{I_{k, q}}^{0}+1\right)^{\frac{1}{2}}\left(S_{I_{k, q}}\right)_{r_{2}} \ldots\left(S_{I_{k, q}}\right)_{r_{p}}\left(H_{I_{k, q}}^{0}+1\right)^{-\frac{1}{2}}\right\| \\
\leq & \left\|V_{I_{k, q}}^{(k, q-1)}\right\|_{H^{0}}\left\|\left(S_{I_{k, q}}\right)_{r_{1}}\left(H_{I_{k, q}}^{0}+1\right)^{\frac{1}{2}}\right\|\left\|\left(H_{I_{k, q}}^{0}+1\right)^{\frac{1}{2}}\left(S_{I_{k, q}}\right)_{r_{2}}\right\| \ldots\left\|\left(S_{I_{k, q}}\right)_{r_{p}}\right\| \\
\leq & c^{p}\left\|V_{I_{k, q}}^{(k, q-1)}\right\|_{H^{0}}\left\|\left(V_{I_{k, q}}^{(k, q-1)}\right)_{r_{1}}\right\|_{H^{0}}\left\|\left(V_{I_{k, q}}^{(k, q-1)}\right)_{r_{2}}\right\|_{H^{0}} \ldots\left\|\left(V_{I_{k, q}}^{(k, q-1)}\right)_{r_{p}}\right\|_{H^{0}}
\end{aligned}
$$

where (A.14) and (A.20) have been used. Putting these terms together, we get the second sum of (A.21).

As for the first sum in (A.8), i.e.,

$$
\sum_{p \geq 2, r_{1} \geq 1 \ldots, r_{p} \geq 1 ; r_{1}+\cdots+r_{p}=j} \frac{1}{p !} \operatorname{ad}\left(S_{I_{k, q}}\right)_{r_{1}}\left(\operatorname{ad}\left(S_{I_{k, q}}\right)_{r_{2}} \ldots\left(\operatorname{ad}\left(S_{I_{k, q}}\right)_{r_{p}}\left(G_{I_{k, q}}\right)\right),\right.
$$

we note that each of its summands is in turn the sum up to a sign of all permutations of

$$
\left(S_{I_{k, q}}\right)_{r_{1}}\left(S_{I_{k, q}}\right)_{r_{2}} \ldots\left(S_{I_{k, q}}\right)_{r_{p-1}}\left[-P_{I_{k, q}}^{+}\left(V_{I_{k, q}}^{(k, q-1)}\right)_{r_{p}} P_{I_{k, q}}^{-}-P_{I_{k, q}}^{-}\left(V_{I_{k, q}}^{(k, q-1)}\right)_{r_{p}} P_{I_{k, q}}^{+}\right]
$$

A very minor variation of the computation above shows that the $\|\cdot\|_{H^{0}}$-norm of the first sum in (A.8) is bounded from above by

$$
\sum_{p=2}^{j} \frac{(2 c)^{p}}{p !} \sum_{r_{1} \geq 1 \ldots, r_{p} \geq 1 ; r_{1}+\cdots+r_{p}=j}\left\|\left(V_{I_{k, q}}^{(k, q-1)}\right)_{r_{1}}\right\|_{H^{0}}\left\|\left(V_{I_{k, q}}^{(k, q-1)}\right)_{r_{2}}\right\|_{H^{0}} \ldots\left\|\left(V_{I_{k, q}}^{(k, q-1)}\right)_{r_{p}}\right\|_{H^{0}}
$$

Henceforth, we closely follow the proof of Theorem 3.2 in [DFFR]; that is, assuming $\left\|V_{I_{k, q}}^{(k, q-1)}\right\|_{H_{0}} \neq$ 0 , we recursively define numbers $B_{j}, j \geq 1$, by the equations

$$
\begin{aligned}
B_{1} & :=\left\|V_{I_{k, q}}^{(k, q-1)}\right\|_{H^{0}}=\left\|\left(V_{I_{k, q}}^{(k, q-1)}\right)_{1}\right\|_{H^{0}}, \\
B_{j} & :=\frac{1}{a} \sum_{k=1}^{j-1} B_{j-k} B_{k}, \quad j \geq 2,
\end{aligned}
$$


with $a>0$ satisfying the relation

$$
e^{2 c a}-1+\left(\frac{e^{2 c a}-2 c a-1}{a}\right)-1=0
$$

Using (A.22), (A.23), (A.21), and an induction, it is not difficult to prove that (see Theorem 3.2 in [DFFR]) for $j \geq 2$

$$
\left\|\left(V_{I_{k, q}}^{(k, q-1)}\right)_{j}\right\|_{H^{0}} \leq B_{j}\left(\frac{e^{2 c a}-2 c a-1}{a}\right)+2\left\|V_{I_{k, q}^{(k, q-1)}}\right\|_{H^{0}} B_{j-1}\left(\frac{e^{2 c a}-1}{a}\right) .
$$

From (A.22) and (A.23) it also follows that

$$
B_{j} \geq \frac{2 B_{j-1}\left\|V_{I_{k, q}}^{(k, q-1)}\right\|_{H^{0}}}{a} \quad \Rightarrow \quad B_{j-1} \leq a \frac{B_{j}}{2 \| V_{I_{k, q}^{(k, q-1)} \|_{H^{0}}}},
$$

which, when combined with (A.25) and (A.24), yields

$$
B_{j} \geq\left\|\left(V_{I_{k, q}}^{(k, q-1)}\right)_{j}\right\|_{H^{0}}
$$

The numbers $B_{j}$ are the Taylor's coefficients of the function

$$
f(x):=\frac{a}{2} \cdot\left(1-\sqrt{1-\left(\frac{4}{a} \cdot\left\|V_{I_{k, q}}^{(k, q-1)}\right\|_{H^{0}}\right) x}\right),
$$

(see [DFFR]). We observe that

$$
\begin{aligned}
\left\|\left(V_{I_{k, q}}^{(k, q-1)}\right)_{j}^{\text {diag }}\right\|_{H^{0}} & =\max \left\{\left\|P_{I_{k, q}}^{(+)}\left(V_{I_{k, q}}^{(k, q-1)}\right)_{j} P_{I_{k, q}}^{(+)}\right\|_{H^{0}},\left\|P_{I_{k, q}}^{(-)}\left(V_{I_{k, q}}^{(k, q-1)}\right)_{j} P_{I_{k, q}}^{(-)}\right\|_{H^{0}}\right\} \\
& =\max _{\#= \pm}\left\|\left(\frac{1}{H_{I_{k, q}}^{0}+1}\right)^{\frac{1}{2}} P_{I_{k, q}}^{(\#)}\left(V_{I_{k, q}}^{(k, q-1)}\right)_{j} P_{I_{k, q}}^{(\#)}\left(\frac{1}{H_{I_{k, q}}^{0}+1}\right)^{\frac{1}{2}}\right\| \\
& =\max _{\#= \pm}\left\|P_{I_{k, q}}^{(\#)}\left(\frac{1}{H_{I_{k, q}}^{0}+1}\right)^{\frac{1}{2}}\left(V_{I_{k, q}}^{(k, q-1)}\right)_{j}\left(\frac{1}{H_{I_{k, q}}^{0}+1}\right)^{\frac{1}{2}} P_{I_{k, q}}^{(\#)}\right\| \\
& \leq\left\|\left(V_{I_{k, q}}^{(k, q-1)}\right)_{j}\right\|_{H^{0}} .
\end{aligned}
$$

Therefore the radius of analyticity, $t_{0}$, of

$$
\sum_{j=1}^{\infty} \tau^{j-1}\left\|\left(V_{I_{k, q}}^{(k, q-1)}\right)_{j}^{\text {diag }}\right\|_{H^{0}}=\frac{1}{\tau}\left(\sum_{j=1}^{\infty} \tau^{j}\left(V_{I_{k, q}}^{(k, q-1)}\right)_{j}^{\text {diag }} \|_{H^{0}}\right)
$$

is bounded below by the radius of analyticity of $\sum_{j=1}^{\infty} x^{j} B_{j}$, i.e.,

$$
t_{0} \geq \frac{a}{4 \| V_{I_{k, q}^{(k, q-1)} \|_{H^{0}}}} \geq \frac{a}{4}
$$

where we have assumed $0<|\tau|<1$ and we have invoked the assumption $\left\|V_{I_{r, i}}^{(k, q-1)}\right\|_{H^{0}} \leq|\tau|^{\frac{r-1}{4}}$. Thanks to the inequality in (A.14), the same bound holds true for the radius of convergence of 
the series $S_{I_{k, q}}:=\sum_{j=1}^{\infty} \tau^{j}\left(S_{I_{k, q}}\right)_{j}$. For $0<|\tau|<1$ and in the interval $\left(0, \frac{a}{8}\right)$, using (A.22) and (A.27), we can estimate

$$
\begin{aligned}
\sum_{j=1}^{\infty}|\tau|^{j-1}\left\|\left(V_{I_{k, q}}^{(k, q-1)}\right)_{j}^{\text {diag }}\right\|_{H^{0}} & \leq \frac{1}{|\tau|} \sum_{j=1}^{\infty}|\tau|^{j} B_{j} \\
& =\frac{1}{|\tau|} \cdot \frac{a}{2} \cdot\left(1-\sqrt{1-\left(\frac{4}{a} \cdot \| V_{\left.I_{k, q}^{(k, q-1)} \|_{H^{0}}\right)|\tau|}\right.}\right) \\
& \leq\left(1+C_{a} \cdot|\tau|\right) \| V_{I_{k, q}^{(k, q-1)} \|_{H^{0}},}
\end{aligned}
$$

for some $a$-dependent constant $C_{a}>0$. Hence the inequality in (A.3) holds true, provided $|\tau|$ is sufficiently small but independent of $N, k$, and $q$. In a similar way, we derive (A.4) and (A.5), using (A.15)-(A.19) and (A.20), respectively.

\section{References}

[BN] S. Bachmann, B. Nachtergaele. On gapped phases with a continuous symmetry and boundary operators J. Stat. Phys. 154(1-2): 91-112 (2014)

[DFF] N. Datta, R. Fernandez, J. Fröhlich. Low-Temperature Phase Diagrams of Quantum Lattice Systems. I. Stability for Quantum Perturbations of Classical Systems with Finitely Many Ground States J. Stat. Phys. 84, 455-534 (1996)

[DFFR] N. Datta, R. Fernandez, J. Fröhlich, L. Rey-Bellet. Low-Temperature Phase Diagrams of Quantum Lattice Systems. II. Convergent Perturbation Expansions and Stability in Systems with Infinite Degeneracy Helvetica Physica Acta 69, 752-820 (1996)

[DFPR] S. Del Vecchio, J. Fröhlich, A. Pizzo, S. Rossi. Lie-Schwinger block-diagonalization and gapped quantum chains with unbounded interactions

[DS] W. De Roeck, M. Salmhofer. Persistence of Exponential Decay and Spectral Gaps for Interacting Fermions Comm. Math. Phys. https://doi.org/10.1007/s00220-018-3211$\mathrm{Z}$

[FFU] R. Fernandez, J. Fröhlich, D. Ueltschi. Mott Transitions in Lattice Boson Models. Comm. Math. Phys. 266, 777-795 (2006)

[FP] J. Fröhlich, A. Pizzo. Lie-Schwinger block-diagonalization and gapped quantum chains https://arxiv.org/abs/1812.02457 (To appear in Comm. Math. Phys.)

[GST] M. Greiter, V. Schnells, R. Thomale. The 1D Ising model and topological order in the Kitaev chain Ann. Phys. 351, 1026-1033 (2014)

[H] M.B. Hastings. The Stability of Free Fermi Hamiltonians https://arxiv.org/abs/1706.02270

[I1] J. Z. Imbrie. Multi-Scale Jacobi Method for Anderson Localization Comm. Math. Phys., 341, 491-521, (2016)

[I2] J. Z. Imbrie. On Many-Body Localization for Quantum Spin Chains J. Stat. Phys., 163, 998-1048, (2016)

[K] Kato. Perturbation of linear operators 
[KST] H. Katsura, D. Schuricht, M. Takahashi . Exact ground states and topological order in interacting Kitaev/Majorana chains Phys. Rev. B 92, 115137 (2015)

[KT] T. Kennedy, H. Tasaki. Hidden symmetry breaking and the Haldane phase in $S=1$ quantum spin chains Comm.. Math. Phys. 147, $431-484$ (1992)

[KU] Kotechy, D. Ueltschi. Effective Interactions Due to Quantum Fluctuations. Comm. Math. Phys. 206, 289-3355 (1999)

[MN] A. Moon, B. Nachtergaele. Stability of Gapped Ground State Phases of Spins and Fermions in One Dimension J. Math. Phys. 59, 091415 (2018)

[NSY] B. Nachtergaele, R. Sims, A. Young. Lieb-Robinson bounds, the spectral flow, and stability of the spectral gap for lattice fermion systems Mathematical Problems in Quantum Physics, pp.93-115

[Y] D.A. Yarotsky. Ground States in Relatively Bounded Quantum Perturbations of Classical Systems Comm. Math. Phys. 261, 799-819 (2006) 